\title{
Lie Algebra Cohomology and $N=2$ SCFT Based on the GKO Construction
}

\author{
Shinobu Hosono ${ }^{1 \star}$ and Akihiro Tsuchiya ${ }^{2}$ \\ ${ }^{1}$ Department of Physics, University of Tokyo, Bunkyo-ku, Tokyo 113, Japan \\ 2 Department of Mathematics, Nagoya University, Nagoya 464, Japan
}

Received May 25, 1990; in revised form June 21, 1990

Dedicated to Professor Noboru Tanaka on his sixtieth birthday

\begin{abstract}
We interpret $N=2$ superconformal field theories (SCFTs) formulated by Kazama and Suzuki via Goddard-Kent-Olive (GKO) construction from a viewpoint of the Lie algebra cohomology theory for the affine Lie algebra. We determine the cohomology group completely in terms of a certain subset of the affine Weyl group. We find that this subset describing the cohomology group can be obtained from its classical counterpart by the action of the Dynkin diagram automorphisms. Some algebra automorphisms of the $N=2$ superconformal algebra are also formulated. Utilizing the algebra automorphisms, we study the field identification problem for the branching coefficient modules in the GKOconstruction. Also the structure of the Poincare polynomial defined for each $N=2$ theory is revealed.
\end{abstract}

\section{Introduction}

Recent progress in two dimensional conformal field theories (CFTs) have revealed rich structures contained in the non-perturbative descriptions of the field theory, or presented us much variety allowed in the field theory. Among them, an important class of the CFTs are the so-called the rational CFTs [Ve, MS1]. The common property we note for the rational CFTs is that these theories can be constructed via Goddard-Kent-Olive (GKO) [GKO] construction using a suitable affine Lie algebra pair $(\hat{\mathfrak{g}}, \hat{\mathfrak{h}})$ with $\hat{\mathfrak{g}} \supseteqq \hat{\mathfrak{h}}$. In this formulation of the CFTs, it is known that a suitable choice of $\hat{\mathrm{g}}$ and $\hat{\mathfrak{h}}$ realizes the CFT with higher symmetry.

Recently Kazama and Suzuki [KS] showed that the $N=2$ superconformal field theory (SCFT) [BFK] [DPZ] [Na] can be constructed through the GKO

* Fellow of the Japan Society for the Promotion of Science 
construction by the pair $(\hat{\mathfrak{g}}, \hat{\mathfrak{h}})$ such that the manifold $G / H$ is the Kähler manifold, where $G$ and $H$ are the finite dimensional Lie groups whose Lie algebras are $g$ and $\mathfrak{h}$, respectively. We note that such a situation is quite reminiscent of the Lie algebra cohomology theory by Kostant [Kos]. In fact, Vafa et al. [LVW] have discussed the super-GKO construction by Kazama-Suzuki from this point of view.

Here, one of the main purposes in this paper is to formulate precisely the $N=2$ super-GKO construction in the context of the affine Lie algebra cohomology theory. We will formulate the theory keeping a parallelism to the Lie algebra cohomology theory by Kostant and its extension by Garland and Lepowski [GL], and then we will make transparent what arises in our case of the affine Lie algebras. One of the characteristic features which we should note for the affine Lie algebra case is that the cohomology theory becomes semi-infinite due to the infinite dimensional structure of the flag manifold. We will see that the semi-infinite structure naturally follows from the viewpoint of the $N=2$ SCFT.

Another interest on the $N=2$ SCFT is in the following observations: Most of the known CFTs are formulated by constructing representations for certain conformal algebras. So it is difficult, in general, to write down a Lagrangian which realizes a given CFT. However, owing to the non-renormalization theorem valid for $N=2$ supersymmetry, we can describe some class of $N=2$ SCFT via the Landau-Ginzburg effective Lagrangian [Zam, Mar, VW]. This occurs for the $N=2$ minimal SCFT $(c<3)$ classified completely in terms of an A-D-E type Lie algebra [CIZ] [Kat]. It has been observed that for each $N=2$ SCFT constructed by A-D-E type modular invariants, there corresponds a Landau-Ginzburg Lagrangian with the superpotential which is also classified via the same A-D-E type Lie algebra in singularity theory [AGV]. Now our second purpose in this article is to investigate the above correspondence between $N=2$ SCFT and the Landau-Ginzburg theory for the general $N=2$ SCFT constructed via the super-GKO construction. For this purpose, a certain Poincaré polynomial will be introduced according to ref. [LVW]. We will reveal the structure of the Poincare polynomial and discuss the fixed point problem noted in ref. [LVW]. Apart from the physical background mentioned above, our curiosity from a mathematical viewpoint is in that the Poincare polynomial thus defined might have an important geometrical meaning associated to the semi-infinite flag manifolds [FF].

The construction of this paper is as follows; first, in Sect. 2, we will present the $N=2$ super-GKO construction in the form which is suitable for our purposes. In Sect. 3, we will interpret the $N=2$ super-GKO construction from the viewpoint of the Lie algebra cohomology theory. The main result there is summarized in Theorem 3.8. Section 4 is devoted to formulate some algebra automorphisms. These algebra automorphisms are used to reveal certain relations among the branching coefficient modules. In Sect. 5, our second purpose mentioned above will be investigated through the definition of the Poincare polynomial. Several results obtained in Sects. 2-4 will be connected in this section. Summary and discussions will be given in the final section.

\section{2. $N=2$ Super-GKO Construction}

In this section we introduce the $N=2$ super-GKO construction formulated by Kazama and Suzuki $[\mathrm{KS}]$ in the form which is suitable for our later arguments. 
To this aim, we must start with establishing our definitions and notations for the (affine) Lie algebra.

Let $\mathrm{g}$ be a simple Lie algebra over $\mathbf{C}$ which has a root space decomposition as;

$$
\mathfrak{g}=\mathfrak{t} \oplus \sum_{\alpha \in \Delta} \mathfrak{g}_{\alpha}
$$

where $\mathrm{t}$ is a fixed Cartan subalgebra and $\Delta=\Delta_{+} \sqcup \Delta_{-}$is the root system. We take the simple system for $\Delta$ as $\Pi=\left\{\alpha_{(1)}, \ldots, \alpha_{(l)}\right\} \quad(l=$ rank $g)$ and normalize the Cartan-Killing form $():, \mathfrak{g} \times \mathfrak{g} \rightarrow \mathbf{C}$ by $(\theta, \theta)=2$ with $\theta$ being the maximal root. Consider a parabolic subalgebra $\mathfrak{p} \subseteq \mathfrak{g}$ which contains the Borel subalgebra $\mathfrak{b} \equiv \mathfrak{t} \oplus \sum_{\alpha \in \Delta_{+}} \mathfrak{g}_{\alpha}$. Such a parabolic subalgebra $\mathfrak{p}$ has a representation as a Lie algebra semi-direct sum with its reductive subalgebra $\mathfrak{h}$ and its radical $\mathfrak{m}_{+}: \mathfrak{p}=\mathfrak{h} \oplus \mathfrak{m}_{+}$. The reductive part $\mathfrak{h}$ of $\mathfrak{p}$ has an orthogonal decomposition with respect to (,) into its abelian part $\mathfrak{h}_{0}$ and the simple parts $\mathfrak{h}_{j}(1 \leqq j \leqq N)$ as

$$
\mathfrak{h}=\mathfrak{h}_{0} \oplus \mathfrak{h}_{1} \oplus \cdots \oplus \mathfrak{h}_{N} .
$$

According to this decomposition of $\mathfrak{h}$, the Cartan subalgebra $\mathfrak{t}$ also decomposes as

$$
\mathrm{t}=\mathrm{t}_{0} \oplus \mathrm{t}_{1} \oplus \cdots \oplus \mathrm{t}_{N} .
$$

In addition, the root system of $\mathfrak{h}$ has the following structure:

$$
\Delta(\mathfrak{h})=\Delta\left(\mathfrak{h}_{1}\right) \sqcup \cdots \sqcup \Delta\left(\mathfrak{h}_{N}\right) \subseteq \Delta .
$$

As a consequence of our definition of $\mathfrak{h}$ and $m_{+}$, we have

$$
\begin{gathered}
\mathfrak{g}=\mathfrak{h} \oplus \mathfrak{m}_{+} \oplus \mathfrak{m}_{-}, \quad \mathfrak{p}=\mathfrak{h} \oplus \mathfrak{m}_{+}, \\
{\left[\mathfrak{h}, \mathfrak{m}_{ \pm}\right] \subseteq \mathfrak{m}_{ \pm}, \quad\left[\mathfrak{m}_{ \pm}, \mathfrak{m}_{ \pm}\right] \subseteq \mathfrak{m}_{ \pm},}
\end{gathered}
$$

where $\mathfrak{m}_{ \pm}=\sum_{\alpha} \mathfrak{g}_{\alpha}$ with $\alpha \in \Delta\left(\mathfrak{m}_{ \pm}\right) \equiv(\Delta \backslash \Delta(\mathfrak{h})) \cap \Delta_{ \pm}$. The direct sum $\mathfrak{p} \equiv \mathfrak{h} \oplus \mathfrak{m}_{+}$is the so-called the parabolic subalgebra of $\mathfrak{g}$.

Let $\left\{J_{\alpha}^{\vee}, H_{\alpha_{(i)}}\right\}_{\alpha \in \Delta, 1 \leqq i \leqq l}$ be the Chevalley $\mathbf{Z}$-form for $\mathfrak{g}$. Then for our later calculations, it is convenient to take our basis of $\mathfrak{g}$ as $\left\{J_{A}\right\}_{A \in \mathscr{A}} \equiv\left\{J_{\alpha}, H_{i}\right\}_{\alpha \in \Delta, 1 \leqq i \leqq l}$, where $J_{\alpha}=\sqrt{\frac{(\alpha, \alpha)}{2}} J_{\alpha}^{\vee}$ and $H_{i}$ 's are orthonormal basis of $\mathfrak{t}_{\mathbf{R}} \equiv \sum_{j=1}^{l} \mathbf{R} H_{\alpha_{(j)}}$ determined so that for each $i$ there exists some $j \in\{0,1, \ldots, N\}$ such that $H_{i} \in \mathfrak{t}_{j}$. We denote the index set $\{1, \ldots, l\}$ for $H \in \mathrm{t}$ as $I=I_{0} \sqcup \cdots \sqcup I_{N}$ with the definition $I_{j} \equiv\left\{i \in I \mid H_{i} \in \mathfrak{t}_{j}\right\}(0 \leqq j \leqq N)$. Then the basis of $\mathfrak{h}_{i}(0 \leqq i \leqq N)$ can be taken as $\left\{J_{a}\right\}_{a \in \mathscr{A}_{j}}$ with $\mathscr{A}_{0}=I_{0}$ and $\mathscr{A}_{i}=\left\{\alpha \in \Delta\left(\mathfrak{h}_{i}\right)\right\} \sqcup I_{i}(1 \leqq i \leqq N)$. Adopting these bases for $\mathfrak{h}_{i}(0 \leqq i \leqq N)$, we naturally introduce a non-degenerate bilinear form $($,$) over$ $\mathfrak{h}_{i}(0 \leqq i \leqq N)$ by restricting the Cartan-Killing form of $\mathfrak{g}$. On the other hand, we may define the normalized Cartan-Killings form $(,)_{i}: \mathfrak{h}_{i} \times \mathfrak{h}_{i} \rightarrow \mathbf{C}$ by requiring $\left(\theta_{i}, \theta_{i}\right)_{i}=2$ for the maximal root $\theta_{i}$ of each simple part $\mathfrak{h}_{i}(1 \leqq i \leqq N)$. Then it is easy to deduce the following relations between the two forms for $0 \leqq i, j \leqq N$ with a convention $\left(\theta_{0}, \theta_{0}\right) \equiv 2$;

$$
\begin{aligned}
& \left(\gamma, \gamma^{\prime}\right)_{i}=\frac{2}{\left(\theta_{i}, \theta_{i}\right)}\left(\gamma, \gamma^{\prime}\right) \quad \text { for } \quad \gamma, \gamma^{\prime} \in \mathrm{t}_{i}^{*}, \\
& \left(J_{a_{i}}, J_{b_{j}}\right)_{i}=\frac{\left(\theta_{i}, \theta_{i}\right)}{2}\left(J_{a_{i}}, J_{b_{j}}\right) \delta_{i j} \quad \text { for } \quad J_{a_{i}} \in \mathfrak{h}_{i}, \quad J_{b_{j}} \in \mathfrak{h}_{j}
\end{aligned}
$$


Under this choice of the basis for $g$ and $\mathfrak{h}_{i}(0 \leqq i \leqq N)$, the anti-C-linear, involutive anti-automorphism $\sigma$ such that $\sigma\left(J_{\alpha}\right)=J_{-\alpha}(\alpha \in \Delta), \sigma\left(H_{i}\right)=H_{i}$ defines the so-called compact real form $\mathfrak{g}_{u} \equiv\{X \in \mathfrak{g} \mid \sigma(X)=-X\}$. In the following, we always assume this real structure when we discuss the hermitian structure on $\mathfrak{g}$ and $\mathfrak{h}_{i}$-modules $(0 \leqq i \leqq N)$. In addition, we introduce a "metric" with respect to this basis by $g_{A B} \equiv\left(J_{A}, J_{B}\right)(A, B \in \mathscr{A})$. This metric will be used to raise or lower the indices of the basis $\left\{J_{A}\right\}_{A \in \mathscr{A}}$.

Here we should describe about the definitions of the affine Lie algebras $\hat{g}$ and $\widehat{\mathfrak{h}}$ (or $\tilde{\mathfrak{g}}$ and $\tilde{\mathfrak{h}}$ ) and their integrable representations. However, since it is rather tiresome, we will give the detailed definitions in the last paragraph of this section. Sophisticated readers are expected to read the last paragraph before proceeding to the next.

Now we can go into the description of the $N=2$ super-GKO construction. The basic ingredients of the $N=2$ super-GKO construction are the $\hat{\mathrm{g}}$-integrable module $L(\hat{\Lambda})$ with highest weight $\hat{\Lambda} \in \hat{P}_{+, k}$ of a fixed level $k$ and the fermion Fock space $\mathscr{F}_{\varepsilon}\left(\varepsilon=0\right.$ or $\frac{1}{2}$ according to the Ramond or the Neveu-Schwartz fermion, respectively). As for the $\hat{\mathfrak{g}}$-module, recall that the integrable $\hat{\mathfrak{g}}$-module $L(\hat{\Lambda})$ admits the unique positive definite hermitian innerproduct $\{$,$\} defined by \{|\hat{\Lambda}\rangle,|\hat{\Lambda}\rangle\}=1$ for the highest vector $|\hat{\Lambda}\rangle \in L(\hat{\Lambda})$ and $J_{A}^{\dagger}(n) \equiv \sigma\left(J_{A}\right)(-n)=J^{A}(-n)$. In the following, we fix this hermitian structure.

We define the Fock space $\mathscr{F}_{\varepsilon}$ in the usual way utilizing the complex fermion oscillators $\psi^{\alpha}(r), \psi_{\alpha}(r) \equiv \psi^{\bar{\alpha}}(r)\left(\alpha \in \Delta\left(\mathrm{m}_{+}\right), \bar{\alpha} \in \Delta\left(\mathrm{m}_{-}\right), \bar{\alpha} \equiv-\alpha, r \in \mathbf{Z}+\varepsilon\right)$ which satisfy the anti-commutation relations as

$$
\begin{aligned}
& \left\{\psi^{\alpha}(r), \psi_{\beta}(s)\right\}=\delta_{\beta}^{\alpha} \delta_{r+s, 0}, \\
& \left\{\psi^{\alpha}(r), \psi^{\beta}(s)\right\}=\left\{\psi_{\alpha}(r), \psi_{\beta}(s)\right\}=0 .
\end{aligned}
$$

The Fock space $\mathscr{F}_{\varepsilon}$ is constructed from the vacuum vector $|0\rangle$ such that

$$
\begin{aligned}
& \psi^{\alpha}(r)|0\rangle=0 \text { for } r>0 \text { and } \alpha \in \Delta\left(\mathrm{m}_{+}\right), \\
& \psi^{\bar{\alpha}}(r)|0\rangle=0 \text { for } r \geqq 0 \text { and } \bar{\alpha} \in \Delta\left(m_{-}\right) .
\end{aligned}
$$

Again we can introduce the unique hermitian innerproduct $\{$,$\} on \mathscr{F}_{\varepsilon}$ by setting $\psi^{\alpha}(r)^{\dagger}=\psi_{\alpha}(-r)$ and $\{|0\rangle,|0\rangle\}=1$.

Now we define the current operators $J_{A}(z)$ and the fermion operators $\psi^{\alpha}(z)$ by the formal Laurent series on a variable $z$;

$$
\begin{aligned}
& J_{A}(z) \equiv \sum_{n \in \mathbf{Z}} J_{A}(n) z^{-n-1}, \\
& \psi^{\alpha}(z) \equiv \sum_{r \in \mathbf{Z}+\varepsilon} \psi^{\alpha}(r) z^{-r-1 / 2} .
\end{aligned}
$$

In order to tame the infinities associated with the infinite summations, we define the following normal orderings;

$$
\begin{gathered}
: \psi^{\alpha}(r) \psi_{\beta}(s):= \begin{cases}\psi^{\alpha}(r) \psi_{\beta}(s) & \left(r \leqq s, \alpha, \beta \in \Delta\left(\mathrm{m}_{+}\right)\right) \\
-\psi_{\beta}(s) \psi^{\alpha}(r) & \left(s<r, \alpha, \beta \in \Delta\left(\mathrm{m}_{+}\right)\right)\end{cases} \\
\text {* }^{*} J_{A}(m) J_{B}(n)_{*}^{*}= \begin{cases}J_{A}(m) J_{B}(n) & (m<n) \\
\frac{1}{2}\left\{J_{A}(m) J_{B}(n)+J_{B}(n) J_{A}(m)\right\} & (m=n) \\
J_{B}(n) J_{A}(m) & (m>n) .\end{cases}
\end{gathered}
$$


The form of the operators $J_{A}(z)$ and $\psi^{\alpha}(z)$ are quite useful in determining operator algebras through the technique of the operator product expansion (OPE) [BPZ] [GO].

In the following, we first construct the $\hat{\mathfrak{h}}$-current operators acting on $\mathscr{F}_{\varepsilon} \otimes L(\hat{\Lambda})$. Then utilizing these operators, we write down the operators which close among themselves as the $N=2 \mathrm{SCA}$. We claim finally that the $N=2 \mathrm{SCA}$ thus constructed does in fact commute with the $\hat{\mathfrak{h}}$-current operators.

Definition 2.1. ( $\left(\hat{\mathfrak{h}}\right.$-current operators): Using the structure constants $f_{A B}{ }^{C}$ of $\mathfrak{g}$ such that $\left[J_{A}, J_{B}\right]=\sum_{C} f_{A B}{ }^{C} J_{C}$, we define the $\hat{\mathfrak{h}}_{i}$-currents $(0 \leqq i \leqq N)$, which act diagonally on $\mathscr{F}_{\varepsilon} \otimes L(\hat{\Lambda})$, by

$$
\hat{J}_{a_{i}}(z) \equiv J_{a_{i}}(z)+J_{a_{i}}^{f}(z) \quad\left(a_{i} \in \mathscr{A}_{i}\right),
$$

with $J_{a_{i}}^{f}(z) \equiv-\sum_{\beta, \gamma \in \Delta\left(\mathfrak{m}_{+}\right)} f_{a_{i} \beta}{ }^{\gamma}: \psi^{\beta}(z) \psi_{\gamma}(z)$ :

Definition 2.2. (Fermion current operators)

$$
\begin{aligned}
& w_{\alpha}(z) \equiv: \psi^{\alpha}(z) \psi_{\alpha}(z): \quad\left(\alpha \in \Delta\left(\mathrm{m}_{+}\right)\right), \\
& J_{f}(z) \equiv \sum_{\alpha \in \Delta\left(\mathfrak{m}_{+}\right)} w_{\alpha}(z) .
\end{aligned}
$$

Using the dual Coxter numbers $g^{*}$ and $h_{i}^{*}$ for $g$ and $\mathfrak{h}_{i}(1 \leqq i \leqq N)$, respectively, we can determine the following OPE relations (, with a convention $h_{0}^{*} \equiv 0$ );

\section{Proposition 2.3.}

1) $\hat{\mathfrak{h}}_{i}$-current operators $(0 \leqq i \leqq N)$ satisfy the following OPE;

$$
\hat{J}_{a_{i}}(z) \hat{J}_{b_{j}}(w) \sim \frac{k_{i}\left(J_{a_{i}}, J_{b_{i}}\right)_{i}}{(z-w)^{2}} \delta_{i j}+\frac{1}{z-w} \sum_{c_{i} \in \mathscr{A}_{i}} f_{a_{i} b_{j}}{ }^{c_{i}} \hat{J}_{c_{i}}(w) \delta_{i j},
$$

with the central charge $k_{i}(0 \leqq i \leqq N)$ given by

$$
k_{i}=\frac{2}{\left(\theta_{i}, \theta_{i}\right)}\left(k+g^{*}-h_{i}^{*} \frac{\left(\theta_{i}, \theta_{i}\right)}{2}\right) \text {. }
$$

2) Fermionic part of the $\hat{\mathfrak{h}}$-current satisfies

$$
\begin{aligned}
J_{a_{i}}^{f}(z) \psi_{\alpha}(w) & \sim \sum_{\beta \in \Delta\left(\mathfrak{m}_{+}\right)} \frac{f_{a_{i} \alpha}^{\beta}}{z-w} \psi_{\beta}(w), \\
J_{a_{i}}^{f}(z) J_{b_{j}}^{f}(w) & \sim \frac{k_{i}^{f}\left(J_{a_{i}}, J_{b_{i}}\right)_{i}}{(z-w)^{2}} \delta_{i j}+\frac{1}{z-w} \sum_{c_{i} \in \mathscr{A}_{i}} f_{a_{i} b_{j}} c_{i} J_{c_{i}}^{f}(w) \delta_{i j},
\end{aligned}
$$

where

$$
k_{i}^{f} \equiv \frac{2}{\left(\theta_{i}, \theta_{i}\right)}\left(g^{*}-h_{i}^{*} \frac{\left(\theta_{i}, \theta_{i}\right)}{2}\right), \quad a_{i} \in \mathscr{A}_{i} \quad \text { and } \quad b_{j} \in \mathscr{A}_{j} .
$$

As we can see above, the action of the current $J_{a_{i}}^{f}(z)$ on the fermion Fock space $\mathscr{F}_{\varepsilon}$ is essentially the adjoint action of $\mathfrak{h}$ on $\mathfrak{m}_{ \pm}:\left[\mathfrak{h}, \mathfrak{m}_{ \pm}\right] \subseteq \mathfrak{m}_{ \pm}$. The central charge $k_{i}^{f}$ is determined by the relation, which follows from

$$
\sum_{B, C \in \mathscr{A}} f_{A}^{B C} f_{B C}^{D}=-(\theta, \theta) g^{*}\left(J_{A}, J^{D}\right)
$$


and

$$
\begin{aligned}
\sum_{b_{i}, c_{i} \in \mathscr{A}_{i}} f_{a_{i}}{ }^{{ }_{i} c_{i}} f_{b_{i} c_{i}}^{d_{i}} & =-\left(\theta_{i}, \theta_{i}\right)_{i} h_{i}^{*}\left(J_{a_{i}}, J^{d_{i}}\right)_{i} \text { for } \mathfrak{h}_{i}{ }^{\prime} \mathrm{s}(1 \leqq i \leqq N), \\
\sum_{\beta, \gamma \in \Delta\left(\mathrm{m}_{+}\right)} f_{a_{i} \beta}{ }^{\gamma} f^{b_{j} \beta} & =-\left(g^{*}-h_{i}^{*} \frac{\left(\theta_{i}, \theta_{i}\right)}{2}\right)\left(J_{a_{i}}, J^{b_{j}}\right) .
\end{aligned}
$$

Using the currents defined above, we can now write down the operators which realize the $N=2$ supersymmetry in $\mathscr{F}_{\varepsilon} \otimes L(\hat{\Lambda})$.

Definition 2.4. $\left(T(z), G^{ \pm}(z), J(z)\right)$,

$$
\begin{aligned}
T(z)= & \frac{1}{2\left(k+g^{*}\right)} \sum_{A \in \mathscr{A}} * J_{A}(z) J^{A}(z)_{*}^{*}+\frac{1}{2} \sum_{\alpha \in \Delta\left(\mathrm{m}_{+}\right)} *^{*} w_{\alpha}(z) w_{\alpha}(z)_{*}^{*} \\
& -\frac{1}{2\left(k+g^{*}\right)} \sum_{i=0}^{N} \sum_{a_{i} \in \mathscr{A}_{i}} *^{*} \hat{J}_{a_{i}}(z) \hat{J}^{a_{i}}(z)_{*}^{*}, \\
G^{+}(z)= & \sqrt{\frac{2}{k+g^{*}}}\left\{\sum_{\alpha \in \Delta\left(\mathrm{m}_{+}\right)} \psi^{\alpha}(z) J_{\alpha}(z)-\frac{1}{2} \sum_{\alpha, \beta, \gamma} f_{\alpha \beta}{ }^{\gamma}: \psi^{\alpha}(z) \psi^{\beta}(z) \psi_{\gamma}(z):\right\}, \\
G^{-}(z)= & \sqrt{\frac{2}{k+g^{*}}}\left\{\sum_{\bar{\alpha} \in \Delta\left(\mathrm{m}_{-}\right)} \psi^{\bar{\alpha}}(z) J_{\bar{\alpha}}(z)-\frac{1}{2} \sum_{\substack{\alpha, \bar{\beta}, \bar{\gamma} \\
\in \Delta\left(\mathrm{m}_{-}\right)}} f_{\bar{\alpha} \bar{\beta}}^{\bar{\gamma}}: \psi^{\bar{\alpha}}(z) \psi^{\bar{\beta}}(z) \psi_{\bar{\gamma}}(z):\right\}, \\
J(z)= & \frac{1}{k+g^{*}} \hat{H}_{2 \rho_{2}}(z)+J_{f}(z), \\
\rho_{2}= & \frac{1}{2} \sum_{\alpha \in \Delta\left(\mathrm{m}_{+}\right)} \alpha .
\end{aligned}
$$

By expanding these operators $T(z), G^{ \pm}(z), J(z)$ on the variable $z$ as $T(z)=$ $\sum_{n} T(n) z^{-n-2}, G^{ \pm}(z)=\sum_{r} G^{ \pm}(r) z^{-r-3 / 2}$ and $J(z)=\sum_{n} J(n) z^{-n-1}$, respectively, we obtain a set of the operators $T(n), G^{ \pm}(r)$ and $J(n)$ with $n \in \mathbf{Z}, r \in \mathbf{Z}+\varepsilon(\varepsilon=0$ or $1 / 2)$. Constructing these definitions of the operators, Kazama and Suzuki found that these operators satisfy the algebra of the $N=2$ SCA. Now we can state their findings for the most general situations: $\mathfrak{g} \supseteqq \mathfrak{h} \supseteqq \mathrm{t}$ :

Theorem 2.5. ( $N=2$ super-GKO construction)

(i) The Virasoro operator $T(n)(n \in \mathbf{Z})$, the super-current $G^{ \pm}(r)(r \in \mathbf{Z}+\varepsilon)$ and the $u(1)$-current $J(n)(n \in \mathbf{Z})$ satisfy the following $N=2$ superconformal algebra (SCA):

For $\varepsilon=\frac{1}{2}(\mathrm{NS}-t y p e)$;

$$
\begin{aligned}
{[T(n), T(m)] } & =(n-m) T(n+m)+\frac{c_{v}}{12}\left(n^{3}-n\right) \delta_{n+m, 0}, \\
{[T(n), J(m)] } & =-m J(n+m), \\
{\left[T(n), G^{ \pm}(r)\right] } & =\left(\frac{n}{2}-r\right) G^{ \pm}(n+r),
\end{aligned}
$$




$$
\begin{aligned}
{[J(n), J(m)] } & =\frac{c_{v}}{3} n \delta_{n+m, 0}, \\
{\left[J(n), G^{ \pm}(r)\right] } & = \pm G^{ \pm}(n+r), \\
\left\{G^{+}(r), G^{-}(s)\right\} & =2 T(r+s)+(r-s) J(r+s)+\frac{c_{v}}{3}\left(r^{2}-\frac{1}{4}\right) \delta_{r+s, 0}, \\
\left\{G^{+}(r), G^{+}(s)\right\} & =\left\{G^{-}(r), G^{-}(s)\right\}=0 .
\end{aligned}
$$

For $\varepsilon=0(R$-type);

$$
\begin{aligned}
{\left[T(n), G^{ \pm}(r)\right] } & =\left(\frac{n}{2} \pm \frac{1}{2}-r\right) G^{ \pm}(n+r), \\
\left\{G^{+}(r), G^{-}(s)\right\} & =2 T(r+s)+(r-s-1) J(r+s)+\frac{c_{v}}{3} r(r-1) \delta_{r+s, 0},
\end{aligned}
$$

and the other (anti-)commutation relations remain the same as in the NS-case.

For both cases, the central charge is given by

$$
\begin{aligned}
c_{v} & =\frac{k \operatorname{dim} \mathfrak{g}}{k+g^{*}}+\operatorname{dim} \mathrm{m}_{+}-\sum_{i=1}^{N} \frac{\left(\theta_{i}, \theta_{i}\right) k_{i} \operatorname{dim} \mathfrak{h}_{i}}{2\left(k+g^{*}\right)}-\operatorname{dim} \mathfrak{h}_{0} \\
& =\frac{3}{k+g^{*}}\left(k \operatorname{dim} \mathrm{m}_{+}-\frac{1}{2} \sum_{\alpha, \beta, \gamma \in \Delta\left(\mathfrak{m}_{+}\right)} f_{\alpha \beta}{ }^{\gamma} f^{\alpha \beta}{ }_{\gamma}\right) .
\end{aligned}
$$

(ii) The above operators of $N=2$ SCA commute with the diagonal action of the $\hat{\mathfrak{h}}$;

$$
\left[T(n), \hat{J}_{a}(m)\right]=\left[G^{ \pm}(r), \hat{J}_{a}(m)\right]=\left[J(n), \hat{J}_{a}(m)\right]=0 .
$$

To prove the above theorem, we must perform somewhat tedious OPE calculations for the operators $(2.15),(2.23)-(2.27)$. For the reader's convenience, we write down a formula which becomes necessary for the calculations. Except for this formula the OPE calculations are straightforward.

Lemma 2.6. The structure constant satisfies

$$
\sum_{i, \alpha} f_{\alpha}^{\alpha i} f_{i \lambda}{ }^{\gamma}=g^{*} g_{\lambda}{ }^{\gamma}-\frac{1}{2} \sum_{\alpha, \beta} f_{\lambda}^{\alpha \beta} f_{\alpha \beta}{ }^{\gamma}+\sum_{\alpha, \bar{\beta}} f_{\lambda}^{\alpha \bar{\beta}} f_{\alpha \bar{\beta}}{ }^{\gamma},
$$

with $\alpha, \beta, \gamma, \lambda \in \Delta\left(\mathrm{m}_{+}\right), \bar{\beta} \equiv-\beta$. The index $i$ is that for the orthonormal basis introduced in $\mathrm{t}$.

Proof. First let us reserve the indices $A, B, a, \alpha, \beta, \gamma$ as $A, B \in \mathscr{A}, a \in \mathscr{A}_{0} \sqcup \cdots \sqcup \mathscr{A}_{N}$ and $\alpha, \beta, \gamma \in \Delta\left(\mathrm{m}_{+}\right)$. In our definition of the invariant, symmetric bilinear form $($,$) the$ structure constant $f_{A B C}$ has a cyclic symmetry as $f_{A B C}=f_{B C A}=f_{C A B}$. Using this property and the Jacobi identity, we can calculate the left-hand side of (2.39) as

$$
\begin{aligned}
\sum_{\alpha, i} f_{\alpha}^{\alpha i} f_{i \lambda}{ }^{\gamma} & =\sum_{\alpha, \boldsymbol{B}} f_{\alpha}^{\alpha \boldsymbol{B}} f_{\boldsymbol{B} \lambda}{ }^{\gamma} \\
& =-\sum_{\boldsymbol{B}, \boldsymbol{A}} f^{\boldsymbol{B} \boldsymbol{A}}{ }_{\lambda} f_{\boldsymbol{B} \boldsymbol{A}}{ }^{\gamma}+\sum_{\boldsymbol{B}, \boldsymbol{a}} f^{\boldsymbol{B} a}{ }_{\lambda} f_{\boldsymbol{B} \boldsymbol{a}}{ }^{\gamma}+2 \sum_{B, \bar{\alpha}} f^{B \bar{\alpha}}{ }_{\lambda} f_{B \bar{\alpha}}{ }^{\gamma} .
\end{aligned}
$$

Noting that the first term of the second line is equal to the 2 nd Casimir of the 
adjoint representation and the relation (2.6) for the other terms, we proceed further as

$$
\text { (l.h.s) }=g^{*}(\theta, \theta) g_{\lambda}{ }^{\gamma}+\sum_{\alpha, \beta} f_{\lambda}^{a \beta} f_{a \beta}{ }^{\gamma}+2 \sum_{\alpha, \bar{\beta}} f_{\lambda \bar{\beta}}^{\alpha \bar{\beta}} f_{\alpha \bar{\beta}}{ }^{\gamma} .
$$

Combining this equality with the relation

$$
\sum_{a, \beta} f_{a \beta}^{\gamma} f_{\lambda}^{a \beta}=-\frac{(\theta, \theta)}{2} g^{*} g_{\lambda}^{\gamma}-\frac{1}{2} \sum_{\alpha, \beta} f_{\alpha \beta}^{\gamma} f_{\lambda}^{\alpha \beta}-\sum_{\alpha, \bar{\beta}} f_{\alpha \bar{\beta}}{ }^{\gamma} f_{\lambda \bar{\beta}}^{\alpha \bar{\beta}},
$$

we obtain the desired result.

The cohomological interpretation for the $N=2$ super-GKO construction introduced above is the main subject in the next section. Before closing this section, we remark on one point that seems to have been overlooked so far in the literature.

Remark. At first sight, it might be seem peculiar that the $N=2$ SCA changes its form depending on whether we take the NS fermion or R fermion. In fact, in the case of $N=1 \mathrm{SCA}$, the algebra (or the OPE) take the same form for both NS and R fermion. We can attribute this peculiarity occurring in the $N=2$ case to the difference of the normal ordering we adopted in (2.13). That is, (2.13) implies, for example, that $: \psi^{\alpha}(0) \psi_{\alpha}(0):=\psi^{\alpha}(0) \psi_{\alpha}(0)$ for $\alpha \in \Delta\left(m_{+}\right)$whereas the conventional normal ordering in the $N=1$ SCFT says $\frac{1}{2}\left\{\psi^{\alpha}(0) \psi_{\alpha}(0)-\psi_{\alpha}(0) \psi^{\alpha}(0)\right\}$. Then the two point functions for the $\mathrm{R}$ fermion are given by

$$
\left\langle\psi_{\alpha}(z) \psi^{\beta}(w)\right\rangle_{R}= \begin{cases}\frac{\sqrt{\frac{z}{w}}}{z-w} \delta_{\alpha}^{\beta} & \text { for our case } \\ \frac{1}{2} \frac{\sqrt{\frac{w}{z}}+\sqrt{\frac{z}{w}}}{z-w} \delta_{\alpha}^{\beta} & \text { for the conventional case. }\end{cases}
$$

This difference of the normal ordering is the origin of the changes such as (2.35) and (2.36). However our choice of the normal ordering (2.13) admits a certain isomorphism between the Fock spaces of the NS-fermion and that for the R-fermion. Owing to this isomorphism we can obtain the (anti-)commutation relations (2.35) and (2.36) for the R-type SCA by simply shifting the argument $r, s \in \mathbf{Z}$ of $G^{+}(r), G^{-}(s)$ in (2.30) and (2.33) to $G^{+}\left(r+\frac{1}{2}\right), G^{-}\left(s+\frac{1}{2}\right)$ and then regarding these shifted operators as $G^{+}(r), G^{-}(s)\left(r, s \in \mathbf{Z}+\frac{1}{2}\right)$ for R-type SCA. It will turn out in Proposition 3.2 that our choice of the definition for the normal ordering (2.13) is crucial for our cohomological interpretations.

- Description of the affine Kac-Moody algebras $\tilde{\mathfrak{g}}$ and $\tilde{\mathfrak{h}}$ :

Let us consider the affine Lie algebra $\hat{\mathfrak{g}}$ defined by $\mathfrak{g} \otimes \mathbf{C}\left[t, t^{-1}\right] \oplus \mathbf{C} c$. The Cartan subalgebra of $\hat{\mathfrak{g}}$ is given by $\hat{\mathfrak{t}}=\mathfrak{t} \oplus \mathbf{C} c$ together with its dual $\mathfrak{t}^{*} \oplus \mathbf{C} \Lambda_{(0)}$. Similarly we define the affine Lie algebra $\hat{\mathfrak{h}}_{i}(0 \leqq i \leqq N)$ by $\mathfrak{h}_{i} \otimes \mathbf{C}\left[t, t^{-1}\right] \oplus \mathbf{C} c_{i}$. The Cartan subalgebra and its dual are fixed by $\hat{\mathrm{t}}_{i} \equiv \mathrm{t}_{i} \oplus \mathbf{C} c_{i}$ and $\mathrm{t}_{i}^{*} \oplus \mathbf{C} \Lambda_{(0) i}$, respectively.

The integrable highest weight modules of $\hat{\mathfrak{g}}$ and $\hat{\mathfrak{h}}$ are parametrized by the dominant integrable weights $\hat{\Lambda} \in \hat{P}_{+, k}$ and $\hat{\xi} \in \hat{P}(\mathfrak{h})_{+, k} \equiv \hat{P}_{+, k_{0}}^{(0)} \oplus \hat{P}_{+, k_{1}}^{(1)} \oplus \cdots \oplus \hat{P}_{+, k_{N}}^{(N)}$, 
respectively. Where the sets of the dominant integrable weights are defined by $\hat{P}_{+, k}=\left\{\bar{\Lambda}+k \Lambda_{(0)} \mid \bar{\Lambda}\right.$ is dominant integral on $\mathfrak{g}$ and $\left.(\bar{\Lambda}, \theta) \leqq k\right\}$ for $\hat{\mathrm{g}}$, and $\hat{P}_{+, k_{i}}^{(i)}=$ $\left\{\bar{\xi}_{i}+k_{i} \Lambda_{(0) i} i \bar{\xi}_{i}\right.$ is dominant integral on $\mathfrak{h}_{i}$ and $\left.\left(\bar{\xi}_{i}, \theta_{i}\right)_{i} \leqq k_{i}\right\}$ for $\hat{\mathfrak{h}}_{i}(1 \leqq i \leqq N)$. As for the abelian part $\hat{\mathfrak{h}}_{0}$, we can introduce the notion of the integral weight in the following way: Consider a "root" lattice $Q_{0} \equiv M \cap \mathrm{t}_{0}^{*}$ with $M$ representing the long root lattice of $\mathfrak{g}$. Then the set of the level $k_{0}$ integral weights for $\hat{\mathfrak{h}}_{0}$ is defined by $\widehat{P}_{+, k_{0}}^{(0)} \equiv\left\{\bar{\xi}_{0}+k_{0} \Lambda_{(0) 0} \mid \bar{\xi}_{0} \in Q_{0}^{\perp}\right\}$ with $Q_{0}^{\perp} \equiv\left\{\lambda \in \mathrm{t}_{0}^{*} \mid(\lambda, \alpha) \in \mathbf{Z}\right.$ for $\left.\forall \alpha \in Q_{0}\right\}$.

The affine Kac-Moody algebra $\tilde{\mathfrak{g}}$ is defined by $\tilde{\mathfrak{g}}=\hat{\mathfrak{g}} \oplus \mathbf{C} d$ as an extension of $\hat{\mathrm{g}}$ by adding the scaling element $d$ which is essentially the Sugawara energy momentum tensor [Sug]. The Cartan subalgebra of $\tilde{\mathfrak{g}}$ and its dual are given by $\tilde{\mathfrak{t}}=\mathrm{t} \oplus \mathbf{C} c \oplus \mathbf{C} d$ and $\tilde{\mathfrak{t}}^{*}=\mathrm{t}^{*} \oplus \mathbf{C} \Lambda_{0} \oplus \mathbf{C} \delta$, respectively. Over this extended algebra $\tilde{\mathrm{g}}$, we can introduce the invariant, symmetric, non-degenerate bilinear form (,) by $\left(J_{A}(n), J_{B}(m)\right)=\delta_{n+m, 0} g_{A B},(c, d)=1$ and $(c, c)=(d, d)=0$, where we have identified the element $J_{A} \otimes t^{n}$ with the current operator $J_{A}(n)$. Similarly we define the affine Kac-Moody algebra $\widetilde{\mathfrak{h}}_{i}(0 \leqq i \leqq N)$ by $\widetilde{\mathfrak{h}}_{i} \equiv \widehat{\mathfrak{h}}_{i} \oplus \mathbf{C} d_{i}$ and its Cartan subalgebras by $\tilde{\mathfrak{t}}_{i} \equiv \mathrm{t}_{i} \oplus \mathbf{C} c_{i} \oplus \mathbf{C} d_{i}, \tilde{\mathfrak{t}}_{i}^{*} \equiv \mathrm{t}_{i}^{*} \oplus \mathbf{C} \Lambda_{(0) i} \oplus \mathbf{C} \delta_{i}$. As is the case for $\mathfrak{h} \cong \mathfrak{g}$, there are two possible Killing forms, the induced form (,) and the normalized form $(,)_{i}$. When we define the induced form through the inclusion map $\widetilde{\mathfrak{h}}_{i} \subseteq \tilde{\mathfrak{g}}(0 \leqq i \leqq N)$ defined by $\left(J_{a_{i}}(n), c_{i}, d_{i}\right) \mapsto\left(J_{a_{i}}(n), \frac{2}{\left(\theta_{i}, \theta_{i}\right)} c, d\right)$, then the relations (2.7) and (2.8) extend to $\tilde{t}_{i}^{*}$ and $\tilde{\mathfrak{h}}_{i}$, respectively.

The simple system for the roots $\tilde{\Delta}$ of $\tilde{\mathrm{g}}$ can be taken as $\tilde{\Pi}=\left\{\alpha_{(0)}, \alpha_{(1)}, \ldots, \alpha_{(l)}\right\}$ with $\alpha_{(0)} \equiv \delta-\theta$. As for the simple systems of $\widetilde{\Delta}\left(\mathfrak{h}_{i}\right){ }^{\prime} s(1 \leqq i \leqq N)$, we take them as $\tilde{\Pi}_{i}=\left\{\alpha_{(0) i}, \alpha_{(1) i}, \ldots, \alpha_{\left(l_{i}\right) i}\right\}$ with $\alpha_{(0) i}=\delta_{i}-\theta_{i}$. Then we denote the root lattice of $\tilde{\mathfrak{g}}$ by $\tilde{Q} \equiv \sum_{i=0}^{l} \mathbf{Z} \alpha_{(i)}$ and $\tilde{Q}_{+} \equiv \sum_{i=0}^{l} \mathbf{Z}_{\geqq 0} \alpha_{(i)}$. Similarly we denote for $\tilde{\mathfrak{h}}_{i}$ as $\tilde{Q}_{i} \equiv \sum_{s=0}^{l_{i}} \mathbf{Z} \alpha_{(s) i}$ and $\tilde{Q}_{i+} \equiv \sum_{s=0}^{l_{i}} \mathbf{Z}_{\geqq 0} \alpha_{(s) i}$

\section{Lie Algebra Cohomology on $\mathscr{F}_{\varepsilon} \otimes L(\hat{\Lambda})$}

In this section we define a chain complex on $\mathscr{F}_{\varepsilon} \otimes L(\hat{\Lambda})$ utilizing the super charges $G^{+}(0)$ (or $G^{+}\left(-\frac{1}{2}\right)$ ) as the cohomology operator. We will determine the elements of the cohomology group completely in terms of the affine Weyl group. It will turn out that this cohomology theory based on the $N=2$ SCA is the affine Lie algebra analogue of the classical Lie algebra cohomology theory. In our formulation, a parallelism to the classical Lie algebra cohomology theory by Kostant [Kos] and its extension by Garland-Lepowski [GL] will be pursued.

(3-1) Chain Complex and Laplacian. We first note that the supercharges $G^{ \pm}(0)$ have a property

$$
\left[J_{f}(0), G^{ \pm}(0)\right]= \pm G^{ \pm}(0)
$$

in addition to $G^{+}(0)^{2}=G^{-}(0)^{2}=0$ and $G^{-}(0)=G^{+}(0)^{\dagger}$. Similar properties also hold for $G^{+}\left(-\frac{1}{2}\right)$ and $G^{-}\left(\frac{1}{2}\right)=G^{+}\left(-\frac{1}{2}\right)^{\dagger}$. It can be easily deduced that these properties define a chain complex on $\mathscr{F}_{\varepsilon} \otimes L(\hat{\Lambda})$;

Definition 3.1. $\left(C_{\varepsilon}^{*}\left(\mathfrak{m}_{+} \otimes \mathbf{C}\left[t, t^{-1}\right], L(\widehat{\Lambda})\right)\right)$. 
We denote as $C_{\varepsilon}^{*}\left(\mathrm{~m}_{+} \otimes \mathbf{C}\left[t, t^{-1}\right], L(\hat{\Lambda})\right)$ the (semi-infinite) co-chain complex on $\mathscr{F}_{\varepsilon} \otimes L(\hat{\Lambda})$ whose coboundary operator is given by $G^{+}(0)$ for $\varepsilon=0$ and $G^{+}\left(-\frac{1}{2}\right)$ for $\varepsilon=1 / 2$. The degree $*$ is determined by integer eigenvalues of the fermion number operator $J_{f}(0)$ for both cases.

It should be remarked that this co-chain complex is semi-infinite reflecting the excitations with infinite fermion or anti-fermion numbers on the Fock space $\mathscr{F}_{\varepsilon}$. Our main subject is to determine the cohomology group $H_{\varepsilon}^{*}\left(\mathrm{~m}_{+} \otimes \mathbf{C}\left[t, t^{-1}\right], L(\hat{\Lambda})\right)$ for the co-chain complex $C_{\varepsilon}^{*}\left(\mathrm{~m}_{+} \otimes \mathbf{C}\left[t, t^{-1}\right], L(\hat{\Lambda})\right)$. Hereafter, we will restrict our arguments to the co-chain complex $C_{\varepsilon}^{*}\left(\mathrm{~m}_{+} \otimes \mathbf{C}\left[t, t^{-1}\right], L(\hat{\Lambda})\right)$ for R-type SCA with omitting the subscript $\varepsilon$. This restriction is allowed since we will see, in next section, that there is an isomorphism between the co-chain complex for R-type SCA and that for NS-type SCA.

For our purposes, we first note that the spaces $H^{*}\left(\mathrm{~m}_{+} \otimes \mathbf{C}\left[t, t^{-1}\right], L(\hat{\Lambda})\right)$ as well as $C^{*}\left(\mathrm{~m}_{+} \otimes \mathbf{C}\left[t, t^{-1}\right], L(\hat{\Lambda})\right)$ admit the diagonal $\hat{\mathfrak{h}}$-action. This is because the supercharge $G^{+}(0)$ commutes with the $\hat{\mathfrak{h}}$-currents $\hat{J}_{a_{i}}(n)(0 \leqq i \leqq N)$ as shown in Theorem 2.5. Owing to this property and the complete reducibility for the integrable modules, we may consider the decomposition $C^{*}\left(\mathrm{~m}_{+} \otimes \mathbf{C}\left[t, t^{-1}\right], L(\hat{\Lambda})\right)$ with respect to the irreducible integrable modules of $\hat{\mathfrak{h}}$ as

$$
C^{*}\left(\mathrm{~m}_{+} \otimes \mathbf{C}\left[t, t^{-1}\right], L(\hat{\Lambda})\right)=\sum_{\hat{\xi} \in \hat{P}(\hat{h})_{+, \mathrm{k}}} C^{*}\left(\mathrm{~m}_{+} \otimes \mathbf{C}\left[t, t^{-1}\right], L(\hat{\Lambda})\right)_{\hat{\xi}},
$$

where $\hat{P}(\mathfrak{h})_{+, \mathbf{k}}$ means the set of the dominant integrable weights for $\hat{\mathfrak{h}}$ with the fixed central charges $\mathbf{k}=\left(k_{0}, \ldots, k_{N}\right)$ given in Proposition 2.3.

On the other hand, let us consider the branching coefficient module $B_{\hat{\Lambda}, \hat{\xi}} \subseteq \mathscr{F}_{0} \otimes$ $L(\hat{\Lambda})$ defined by

$$
\begin{aligned}
B_{\hat{\Lambda}, \hat{\xi}} \equiv\left\{v \in \mathscr{F}_{0} \otimes L(\hat{\Lambda}) \mid \hat{J}(n) v=0(J \in \mathfrak{h}, n>0), \hat{J}_{\alpha}(0) v\right. & =0\left(\alpha \in \Delta(\mathfrak{h})_{+}\right), \\
\hat{H}(0) v & =\langle H, \widehat{\xi}\rangle v(H \in \mathfrak{t})\} .
\end{aligned}
$$

The analysis given in a previous section tells us that the $N=2$ SCA (2.28)-(2.36) acts on this branching coefficient modules $B_{\hat{\Lambda}, \hat{\xi}}$ in $\mathscr{F}_{0} \otimes L(\hat{\Lambda})$. Then we see that there is an isomorphism (see ref. [TK] or Eq. (4.24) in the next section for detailed constructions)

$$
C^{*}\left(\mathrm{~m}_{+} \otimes \mathbf{C}\left[t, t^{-1}\right], L(\hat{\Lambda})\right)_{\hat{\xi}} \cong B_{\hat{\Lambda}, \hat{\xi}} \otimes L_{\mathfrak{h}}(\hat{\xi}),
$$

as an $N=2 \mathrm{SCA} \otimes \hat{\mathfrak{h}}$-module.

Now let us evaluate the Laplacian, which we read from the $N=2$ SCA (2.37) as

$$
\left\{G^{+}(0), G^{-}(0)\right\}=2 T(0)-J(0) \text {, }
$$

on each branching coefficient module $B_{\hat{\Lambda}, \hat{\xi}}$. To this aim, we first decompose the module $B_{\hat{\Lambda}, \hat{\xi}}$ into the eigenspaces of the operator

$$
\hat{N} \equiv T_{\mathrm{g}}(0)+T_{f}(0)-\frac{1}{2} J_{f}(0)-\frac{(\bar{\Lambda}, \bar{\Lambda}+2 \rho)}{2\left(k+g^{*}\right)}
$$

which acts on the module $B_{\hat{\Lambda}, \hat{\xi}}$. The fact that this operator acts on the module $B_{\hat{\Lambda}, \hat{\xi}}$ follows from the following commutation relation;

$$
\left[\hat{N}, \hat{J}_{a}(n)\right]=\left[\hat{T}_{\mathrm{h}}(0), \hat{J}_{a}(n)\right]=-n \hat{J}_{a}(n) .
$$

Above we have used the notations $T_{\mathrm{g}}(0), T_{f}(0)$ and $\widehat{T}_{\mathrm{h}}(0)$ for the Sugawara operators 
for $\hat{\mathrm{g}}$-curents, fermion currents and $\hat{\mathfrak{h}}$-currents, respectively. Since $\left[T_{f}(0)-\frac{1}{2} J_{f}(0)\right.$, $\left.\psi^{\alpha}(-r)\right]=r \psi^{\alpha}(-r)\left(\alpha \in \Delta\left(\mathfrak{m}_{+}\right) \sqcup \Delta\left(\mathfrak{m}_{-}\right), r \in \mathbf{Z}\right)$, under the definition of the normal ordering (2.14) for R-type fermion, the eigenvalues of the decomposition becomes (non-negative) integers. Thus we have the desired decomposition as

$$
B_{\hat{\Lambda}, \hat{\xi}}=\sum_{N \in \mathbf{Z} \geqq 0} B_{\hat{\Lambda}, \hat{\xi}, N} .
$$

Note that the above decomposition is compatible with our calculation of the cohomology since the operator we have used commutes with the Laplacian.

Proposition 3.2. The Laplacian acts as scalar on $B_{\hat{A}, \hat{\xi}, N}$;

$$
\left.\left\{G^{+}(0), G^{-}(0)\right\}\right|_{B_{\hat{\lambda}, \hat{\xi}, N}}=\frac{1}{k+g^{*}}\left\{|\hat{\Lambda}+\hat{\rho}|^{2}-\left|\hat{\xi}^{\prime}+\hat{\rho}\right|^{2}\right\} \text { id. }
$$

where $\hat{\xi}^{\prime}=k \Lambda_{0}+\bar{\xi}-N \delta, \hat{\rho}=g^{*} \Lambda_{0}+\frac{1}{2} \sum_{\alpha \in \Delta_{+}} \alpha$ and $\bar{\xi}$ means the classical part of $\hat{\xi}$. Proof. Recall that the Laplacian is given by $2 T(0)-J(0)=2 \hat{N}+\frac{(\bar{\Lambda}, \bar{\Lambda}+2 \rho)}{k+g^{*}}-$ $2 \hat{T}_{\mathfrak{b}}(0)-\frac{2}{k+g^{*}} \hat{H}_{\rho_{2}}(0)$. Then it is an easy consequence of our definitions that the Laplacian acts on the space $B_{\hat{\Lambda}, \hat{\xi}, N}$ as $\left.\left\{G^{+}(0), G^{-}(0)\right\}\right|_{B_{\hat{\Lambda}, \bar{\xi}, N}}=2\left\{N+\frac{(\bar{\Lambda}, \bar{\Lambda}+2 \rho)}{2\left(k+g^{*}\right)}-\right.$ $\left.\frac{\left(\bar{\xi}, \bar{\xi}+2 \rho_{1}\right)}{2\left(k+g^{*}\right)}-\frac{\left(\rho_{2}, \bar{\xi}\right)}{k+g^{*}}\right\}$ id. with $\rho_{1} \equiv \frac{1}{2} \sum_{\gamma \in \Delta_{+}(\mathfrak{l})} \gamma$. And we immediately obtain the results (3.8).

(3-2) Elements of the Cohomology Group. As noted previously, the cohomology group $H^{*}\left(\mathfrak{m}_{+} \otimes \mathbf{C}\left[t, t^{-1}\right], L(\hat{\Lambda})\right)$ for the cochain complex $C^{*}\left(\mathfrak{m}_{+} \otimes \mathbf{C}\left[t, t^{-1}\right], L(\hat{\Lambda})\right)$ becomes naturally a $\hat{\mathfrak{h}}$-module. So we obtain a decomposition with respect to the integrable irreducible $\hat{\mathfrak{h}}$-modules, corresponding to (3.2), as

$$
H^{*}\left(\mathfrak{m}_{+} \otimes \mathbf{C}\left[t, t^{-1}\right], L(\hat{\Lambda})\right)=\sum_{\hat{\xi} \in \hat{P}(())+, k} H^{*}\left(\mathfrak{m}_{+} \otimes \mathbf{C}\left[t, t^{-1}\right], L(\hat{\Lambda})\right)_{\hat{\xi}} .
$$

On the other hand, according to the Hodge theory, we can characterize the cohomology elements as the zeros of the Laplacian:

$$
\begin{aligned}
& H^{*}\left(\mathfrak{m}_{+} \otimes \mathbf{C}\left[t, t^{-1}\right], L(\hat{\Lambda})\right)_{\hat{\xi}} \\
& \quad \cong \mathbf{H}^{*}\left(\mathfrak{m}_{+} \otimes \mathbf{C}\left[t, t^{-1}\right], L(\hat{\Lambda})\right)_{\hat{\xi}} \\
& \quad \equiv\left\{v \in C^{*}\left(\mathfrak{m}_{+} \otimes \mathbf{C}\left[t, t^{-1}\right], L(\hat{\Lambda})\right)_{\hat{\xi}} \mid G^{+}(0) v=G^{-}(0) v=0\right\} .
\end{aligned}
$$

Because the cohomology operator $G^{ \pm}(0)$ commutes with the $\hat{\mathfrak{h}}$-currents, the harmonic cocycles in (3.10), which constitutes the $\hat{\mathfrak{h}}$-modules, can be represented by the elements $v \in B_{\hat{\lambda}, \hat{\xi}}$ satisfying $\left\{G^{+}(0), G^{-}(0)\right\} v=0$. On the other hand we know from Proposition 3.2 that the Laplacian operator acts as a scalar when restricted on the module $B_{\hat{\lambda}, \hat{\xi}}$. Therefore we can completely characterize the harmonic cocycles in $C^{*}\left(\mathfrak{m}_{+} \otimes \mathbf{C}\left[t, t^{-1}\right], L(\hat{\Lambda})\right)$ through the zeros of the right-hand side of (3.8) in Proposition 3.2. This is our strategy for determining the cohomology elements of $H^{*}\left(\mathfrak{m}_{+} \otimes \mathbf{C}\left[t, t^{-1}\right], L(\hat{\Lambda})\right)$.

To perform the above mentioned program, we must start with summarizing some known properties of the affine Weyl group. 
The affine Weyl group $\tilde{W}$ of $\hat{\mathrm{g}}$ is the subgroup of $G L\left(\tilde{\mathrm{t}}^{*}\right)$ generated by the simple reflections $\left\{r_{0}, r_{1}, \ldots, r_{l}\right\}$ with

$$
r_{i}(\lambda)=\lambda-\left(\lambda, \alpha_{(i)}^{\vee}\right) \alpha_{(i)} \quad\left(\alpha_{(i)}^{\vee} \equiv \frac{2}{\left(\alpha_{(i)}, \alpha_{(i)}\right)} \alpha_{(i)}, \lambda \in \tilde{\mathfrak{t}}^{*}, 0 \leqq i \leqq l\right) .
$$

Introduce the translation $t_{\alpha} \in G L\left(\tilde{\mathrm{t}}^{*}\right)\left(\alpha \in \mathrm{t}^{*}\right)$ by

$$
t_{\alpha}(\lambda)=\lambda+(\lambda, \delta) \alpha-\left[(\lambda, \alpha)+\frac{1}{2}(\alpha, \alpha)(\lambda, \delta)\right] \delta, \quad\left(\lambda \in \tilde{\mathfrak{t}}^{*}\right) .
$$

If we define the translation group $T \equiv\left\{t_{\alpha} \mid \alpha \in M\right\}$, then the affine Weyl group $\tilde{W}$ can be expressed as $\tilde{W}=T \ltimes W$. (The long root lattice $M$ coincides with the coroot lattice defined by $Q^{\vee} \equiv \sum_{i=1}^{l} \mathbf{Z} \alpha_{(i)}^{\vee}$ since $(\theta, \theta)=2$.)

Now let us consider a subset $\tilde{W}^{1} \subseteq \tilde{W}$, which will play an important role in the following arguments, by

$$
\tilde{W}^{1} \equiv\left\{\tilde{\omega} \in \tilde{W} \mid \tilde{\omega}\left(\tilde{\Delta}_{-}\right) \cap \tilde{\Delta}_{+} \subseteq \tilde{\Delta}(\mathfrak{m})_{+}\right\},
$$

where $\tilde{\Delta}(\mathfrak{m})_{+}=\left\{\beta+n \delta \mid \beta \in \Delta\left(\mathrm{m}_{+}\right), n \in \mathbf{Z}_{\geqq 0}\right\} \sqcup\left\{\bar{\beta}+n \delta \mid \bar{\beta} \in \Delta\left(\mathrm{m}_{-}\right), n \in \mathbf{Z}_{>0}\right\}$. For each $\tilde{\omega} \in \tilde{W}$, let $\Phi_{\tilde{\omega}} \equiv \tilde{\omega}\left(\tilde{\Delta}_{-}\right) \cap \tilde{\Delta}_{+}$and $\left\langle\Phi_{\tilde{\omega}}\right\rangle \equiv \sum_{\varphi \in \Phi_{\tilde{\omega}}} \varphi \in \tilde{Q}_{+}$. We denote the order of the subset $\Phi_{\tilde{\omega}}$ as $n(\tilde{\omega})$ and the length of the element $\tilde{\omega}$ as $l(\tilde{\omega})$. Then the following relations are known to hold [IM, GL];

$$
\begin{aligned}
\text { i) } & \left\langle\Phi_{\tilde{\omega}}\right\rangle=\hat{\rho}-\tilde{\omega} \hat{\rho}, \\
\text { ii) } & \left\langle\Phi_{\tilde{\omega}}\right\rangle=\left\langle\Phi_{\tilde{\sigma}}\right\rangle \Leftrightarrow \tilde{\omega}=\tilde{\sigma}, \\
\text { iii) } & n(\tilde{\omega})=l(\tilde{\omega}),
\end{aligned}
$$

In addition to these properties, the following is also well-known and is an important property;

$$
\text { iv) } r_{i}\left(\tilde{\Delta}_{+} \backslash\left\{\alpha_{(i)}\right\}\right)=\tilde{\Delta}_{+} \backslash\left\{\alpha_{(i)}\right\} \quad(i=0,1, \ldots, l) .
$$

We shall study in detail the set $\tilde{W}^{1}$ in the latter half of this section. In the following we investigate the zeros of the Laplacian (3.8) following refs. [Kos, GL].

Lemma 3.3. Let $T_{\hat{\Lambda}} \subseteq \hat{\Lambda}-\tilde{Q}_{+}$be the $\tilde{W}$-invariant subset of $\tilde{\mathfrak{t}}^{*}$ with $\hat{\Lambda} \in \hat{P}_{+}$. Then for arbitrary $\tilde{\mu} \in T_{\hat{\Lambda}}$, there exists $\tilde{\omega} \in \tilde{W}$ such that $\tilde{\omega} \tilde{\mu} \in \tilde{P}_{+}$.

Proof. For arbitrary $\tilde{\mu} \in T_{\hat{\Lambda}}$, take an element $\tilde{\omega} \in \tilde{W}$ so that in the expression $\tilde{\omega} \tilde{\mu}=\hat{\Lambda}-\sum_{i=0}^{l} n_{i} \alpha_{(i)}\left(n_{i} \in \mathbf{Z}_{\geqq 0}\right)$ the sum $\sum_{i=0}^{l} n_{i}$ is minimum. Then $\tilde{\omega} \tilde{\mu} \in \tilde{P}_{+}$. This is because if $\left(\tilde{\omega} \tilde{\mu}, \alpha_{(j)}^{\vee}\right)<0$ for some $j$ then $r_{j} \tilde{\omega} \tilde{\mu}=\tilde{\omega} \tilde{\mu}-\left(\tilde{\omega} \tilde{\mu}, \alpha_{(j)}^{\vee}\right) \alpha_{(j)}$ can be written as $\hat{\Lambda}-\sum_{i=0}^{l} m_{i} \alpha_{(i)}$ with $\sum_{i=0}^{l} m_{i}<\sum_{i=0}^{l} n_{i}$, which is a contradiction.

Lemma 3.4. Let $\hat{\Lambda}_{1}, \hat{\Lambda}_{2} \in \hat{P}_{+}$. Consider $\tilde{\mu}_{1}, \tilde{\mu}_{2} \in \tilde{\mathfrak{t}}^{*}$ which belong to the $\tilde{W}$-invariant subset $T_{\hat{\Lambda}_{1}} \subseteq \hat{\Lambda}_{1}-\widetilde{Q}_{+}$and $T_{\hat{\Lambda}_{2}} \subseteq \hat{\Lambda}_{2}-\widetilde{Q}_{+}$, respectively. Then

$$
\left|\hat{\Lambda}_{1}+\hat{\Lambda}_{2}\right| \geqq\left|\tilde{\mu}_{1}+\tilde{\mu}_{2}\right| \text {. }
$$

The equality holds if and only if there exists $\tilde{\omega} \in \tilde{W}$ such that $\tilde{\omega} \hat{\Lambda}_{1}=\tilde{\mu}_{1}$ and $\tilde{\omega} \hat{\Lambda}_{2}=\tilde{\mu}_{2}$. Such $\tilde{\omega}$ is determined uniquely if $\hat{\Lambda}_{1}$ or $\hat{\Lambda}_{2}$ is regular. 
Proof. Due to Lemma 3.3, we can determine $\tilde{\tau} \in \tilde{W}$ so that $\tilde{\tau}\left(\tilde{\mu}_{1}+\tilde{\mu}_{2}\right) \in \tilde{P}_{+}$. Because the sets of weights $T_{\hat{\Lambda}_{i}}(i=1,2)$ are $\tilde{W}$-invariant, we have $\hat{\psi}_{i} \equiv \hat{\Lambda}_{i}-\tilde{\tau} \tilde{\mu}_{i} \in \tilde{Q}_{+}$for $u=1,2$. Then we can write the square of the left-hand side of (3.18) as

$$
\left|\hat{\Lambda}_{1}+\hat{\Lambda}_{2}\right|^{2}=\left|\tilde{\mu}_{1}+\tilde{\mu}_{2}\right|^{2}+\left|\hat{\psi}_{1}+\hat{\psi}_{2}\right|^{2}+2\left(\hat{\psi}_{1}+\hat{\psi}_{2}, \tilde{\tau}\left(\tilde{\mu}_{1}+\tilde{\mu}_{2}\right)\right) .
$$

Since $\tilde{\tau}\left(\tilde{\mu}_{1}+\tilde{\mu}_{1}\right) \in \tilde{P}_{+}$, the desired inequality immediately follows. In addition the equality for (3.18) holds if and only if $\hat{\psi}_{1}+\hat{\psi}_{2}=0$. In this case we must have $\hat{\Lambda}_{1}=\tilde{\tau} \tilde{\mu}_{1}$ and $\hat{\Lambda}_{2}=\tilde{\tau} \tilde{\mu}_{2}$ since $\hat{\psi}_{1}, \hat{\psi}_{2} \in \tilde{Q}_{+}$.

As for the uniqueness when $\hat{\Lambda}_{1}$ or $\hat{\Lambda}_{2}$ is regular, assume $\tilde{\tau}_{1}, \tilde{\tau}_{2} \in \tilde{W}$ satisfy $\hat{\Lambda}_{i}=\tilde{\tau}_{1} \tilde{\mu}_{i}$ and $\hat{\Lambda}_{i}=\tilde{\tau}_{2} \tilde{\mu}_{i}$ for $i=1,2$. Then $\tilde{\tau}_{1}$ must be eqal to $\tilde{\tau}_{2}$ since $\tilde{\tau}_{1}^{-1} \tilde{\tau}_{2} \tilde{\Lambda}_{i}=\tilde{\Lambda}_{i}(i=1,2)$ implies $\tilde{\tau}_{1}^{-1} \tilde{\tau}_{2}=1$ for the regular element $\hat{\Lambda}_{1}$ or $\hat{\Lambda}_{2}$.

Lemma 3.5. The subset $T_{\hat{\rho}}^{\prime} \subseteq \tilde{\mathrm{t}}^{*}$ which consists of the element of the form $\hat{\rho}-\langle\Phi\rangle$ with $\Phi$ being a finite subset of $\Delta_{+}$is $\tilde{W}$-invariant.

Proof. Consider the action $r_{i}(i=0, \ldots, l)$ on $\hat{\rho}-\langle\Phi\rangle \in T_{\hat{\rho}}^{\prime}$. If $\alpha_{(i)} \notin \Phi$ then $r_{i}$ $(\hat{\rho}-\langle\Phi\rangle)=\left(r_{i} \hat{\rho}-\hat{\rho}\right)+\hat{\rho}-r_{i}\langle\Phi\rangle=\hat{\rho}-r_{i}\langle\Phi\rangle-\left\langle\Phi_{i_{i}}\right\rangle$. Owing to the property iv) in (3.17), we see that both $r_{i}\langle\Phi\rangle$ and $\left\langle\Phi_{r_{i}}\right\rangle=\alpha_{(i)}$ belongs to the set $\tilde{\Delta}_{+}$. If $\alpha_{(i)} \in \Phi$, then $r_{i}\left(\hat{\rho}-\left\langle\Phi \backslash\left\{\alpha_{(i)}\right\}\right\rangle-\alpha_{(i)}\right)=\left(r_{i} \hat{\rho}-\hat{\rho}\right)+\hat{\rho}-r_{i}\left\langle\Phi \backslash\left\{\alpha_{(i)}\right\}\right\rangle+\alpha_{(i)}=\hat{\rho}-r_{i}\left\langle\Phi \backslash\left\{\alpha_{(i)}\right\}\right\rangle$. Again owing to the property iv) in (3.17), see that $r_{i}\left\langle\Phi \backslash\left\{\alpha_{(i)}\right\}\right\rangle \in \tilde{\Delta}_{+}$. Thus we obtain $r_{i}(\hat{\rho}-\langle\Phi\rangle) \in T_{\hat{\rho}}^{\prime}$ and conclude that $T_{\hat{\rho}}^{\prime}$ is $\tilde{W}$-invariant.

Lemma 3.6. Let $\tilde{\omega} \in \tilde{W}, \Phi \subseteq \tilde{\Delta}_{+}$such that $|\Phi|<\infty$, then

$$
\langle\Phi\rangle=\left\langle\Phi_{\tilde{\omega}}\right\rangle \Leftrightarrow \Phi=\Phi_{\tilde{\omega}}(\tilde{\omega} \in \tilde{W}) .
$$

In Particular $\Phi=\Phi_{\tilde{\omega}}$ consists of real roots.

Proof. Let $\mu_{1}, \ldots, \mu_{m} \in \tilde{\Delta}_{+}$be the distinct elements of $\Phi$, and $v_{1}, \ldots, v_{n} \in \tilde{\Delta}_{+}$be those of $\Phi_{\tilde{\omega}}$. (Then $v_{k}$ 's are all real roots.) When $n=0$, the result it is clear, so we assume $n>0$. Since $\tilde{\omega}^{-1} \Phi_{\tilde{\omega}} \leqq \tilde{\Delta}_{-}$, there must exist some element $\mu_{i}(1 \leqq i \leqq m)$ such that $\tilde{\omega}^{-1} \mu_{i} \in \Delta_{-}$. This means that $\mu_{i}=v_{j}$ for some $j(1 \leqq j \leqq n)$, and $\left\langle\Phi_{\omega} \backslash\left\{v_{j}\right\}\right\rangle=\left\langle\Phi \backslash\left\{\mu_{i}\right\}\right\rangle$. If $n=1$, we accomplish the proof. When $n>1$, we have $\omega^{-1}\left(\Phi_{\omega} \backslash\left\{v_{j}\right\}\right) \subseteq \tilde{\Delta}_{-}$and so there must exist some element $\mu_{i^{\prime}}\left(i \neq i^{\prime}\right)$ such that $\omega^{-1} \mu_{i^{\prime}} \in \widetilde{\Delta}_{-}$. This means that $\mu_{i^{\prime}}=v_{j^{\prime}}$ for some $j^{\prime}(\neq j)$ and $\left\langle\Phi_{\omega} \backslash\left\{v_{j}, v_{j^{\prime}}\right\}\right\rangle=\left\langle\Phi \backslash\left\{\mu_{i}, \mu_{i^{\prime}}\right\}\right\rangle$. Repeated arguments prove the result.

Now let us consider the weight space decomposition of $\mathscr{F}_{0} \otimes L(\hat{\Lambda})$ with respect to the commuting operators $\left(\hat{H}_{i}(0), c,-\hat{N}\right)(1 \leqq i \leqq l)$, where the classical part $\hat{H}_{i}(0)$ is that of the $\hat{\mathfrak{h}}$-currents defined by (2.16). Then we deduce, from the definition of the $\hat{\mathfrak{h}}$-current operators, that the set of weights, which we denote as $D_{\hat{\Lambda}}$, consist of the elements such that $\tilde{\zeta}=-\langle\Phi\rangle+\tilde{v}$ with $\Phi \subseteq \tilde{\Delta}(\mathrm{m})_{+}$and $\tilde{v} \in P(\hat{\Lambda})$. Where we have denoted as $P(\hat{\Lambda})$ the set of weights contained in the integrable $\hat{\mathrm{g}}$-module $L(\hat{\Lambda})$. Needless to say, this subset $P(\hat{\Lambda}) \in \tilde{\mathfrak{t}}^{*}$ is $\tilde{W}$-invariant.

\section{Proposition 3.7.}

i) For arbitrary $\tilde{\zeta} \in D_{\hat{\Lambda}}$, there is an inequality;

$$
|\hat{\rho}+\hat{\Lambda}| \geqq|\hat{\rho}+\tilde{\zeta}|
$$

ii) All the weights $\tilde{\zeta} \in D_{\hat{\Lambda}}$ which satisfy the equality for (3.20) are expressed uniquely 
with some element $\tilde{\omega} \in \tilde{W}^{1}$ as

$$
\xi_{\tilde{\omega}} \equiv \tilde{\omega}(\hat{\rho}+\hat{\Lambda})-\hat{\rho} .
$$

iii) The map $\tilde{\omega} \mapsto \xi_{\tilde{\omega}}$ defines a bijection between the subset $\tilde{W}^{1}$ and the set of weights which satisfy the equality for (3.20).

iv) The multiplicity of the weight $\xi_{\tilde{\omega}} \in D_{\hat{\Lambda}}$ is one.

v) The weight vector $v\left(\xi_{\tilde{\omega}}\right) \in \mathscr{F}_{0} \otimes L(\hat{\Lambda})\left(\tilde{\omega} \in \tilde{W}^{1}\right)$ is the highest weight vector with respect to the diagonal action $\hat{\mathfrak{h}}$.

\section{Proof.}

i), ii) Since $\tilde{\zeta} \in D_{\hat{\Lambda}}$ can be written as $\tilde{\zeta}=-\langle\Phi\rangle+\tilde{v}$ with $\Phi \subseteq \tilde{\Delta}(m)+$ and $\tilde{v} \in P(\hat{\Lambda})$, we can write $\hat{\rho}+\widetilde{\zeta}=\tilde{f}+\tilde{v}$ by defining $\tilde{f} \equiv \hat{\rho}-\langle\Phi\rangle \in T_{\hat{\rho}}^{\prime}$. Then from Lemma 3.4, we obtain

$$
|\hat{\rho}+\hat{\Lambda}| \geqq|\tilde{f}+\tilde{v}|=|\hat{\rho}+\tilde{\zeta}| .
$$

When the equality holds, there exists a unique element $\tilde{\omega} \in \tilde{W}$ such that $\tilde{f}=\tilde{\omega} \hat{\rho}$, $\tilde{v}=\tilde{\omega} \hat{\Lambda}$ (i.e., $\tilde{\zeta}=\xi_{\tilde{\omega}}$ ) owing to Lemma 3.4 and the fact that $\hat{\rho}$ is regular. Then the first equality $\tilde{f}=\tilde{\omega} \tilde{\rho}$, which means $\langle\Phi\rangle=\hat{\rho}-\tilde{\omega} \hat{\rho}$, implies $\Phi=\Phi_{\tilde{\omega}}$ due to Lemma 3.6. Since $\Phi \subseteq \tilde{\Delta}(\mathrm{m})_{+}$, the element $\tilde{\omega}$ belongs to the subset $\tilde{W}^{1} \subseteq \tilde{W}$.

iii) We easily see by definitions that, for arbitrary $\tilde{\omega} \in \tilde{W}^{1}, \xi_{\tilde{\omega}}$ belongs to the weight set $D_{\hat{\Lambda}}$. If $\xi_{\tilde{\omega}}=\xi_{\tilde{\omega}^{\prime}}\left(\tilde{\omega}, \tilde{\omega}^{\prime} \in \tilde{W}^{1}\right)$ then $\tilde{\omega}=\tilde{\omega}^{\prime}$ because $\hat{\rho}+\hat{\Lambda} \in \hat{P}_{+}$is regular. These facts together with ii) tell us the bijectivity of the map.

iv) Assume that the weight $\xi_{\tilde{\omega}} \in D_{\hat{\Lambda}}$ is written as

$$
\xi_{\tilde{\omega}}=-\langle\Phi\rangle+\tilde{\mu}, \quad \tilde{\mu} \in P(\hat{\Lambda}) \quad \Phi \subseteq \tilde{\Lambda}(\mathfrak{m})_{+},
$$

then it follows that $\tilde{\omega}(\hat{\rho}+\hat{\Lambda})=\hat{\rho}-\langle\Phi\rangle+\tilde{\mu}$ and $|\hat{\rho}+\hat{\Lambda}|=|\hat{\rho}-\langle\Phi\rangle+\tilde{\mu}|$. Since $\hat{\rho}-\langle\Phi\rangle \in T_{\hat{\rho}}^{\prime}$ and $\tilde{\mu} \in P(\hat{\Lambda})$, we can determine, according to Lemma 3.4, a unique element $\tilde{\sigma} \in \tilde{W}$ such that

$$
\hat{\rho}-\langle\Phi\rangle=\tilde{\sigma} \hat{\rho}, \quad \tilde{\mu}=\tilde{\sigma} \hat{\Lambda} .
$$

This implies $\xi_{\tilde{\omega}}=\xi_{\tilde{\sigma}}$ and, in turn, $\tilde{\sigma}=\tilde{\omega}$. Noting $\langle\Phi\rangle=\left\langle\Phi_{\tilde{\sigma}}\right\rangle=\left\langle\Phi_{\tilde{\omega}}\right\rangle$ and Lemma 3.6, we finally obtain $\Phi=\Phi_{\tilde{\omega}}$. Thus we conclude that the multiplicity of the weight vector $\xi_{\tilde{\omega}}$ is one.

v) Consider the weights of the vectors $\hat{J}_{a_{i}}(n) v\left(\xi_{\tilde{\omega}}\right)\left(a_{i} \in \mathscr{A}_{i}, n>0,0 \leqq i \leqq N\right)$ and $\widehat{J}_{\alpha}(0) v\left(\xi_{\tilde{\omega}}\right)\left(\alpha \in \Delta(\mathfrak{h})_{+}\right)$with respect to the operators $\left(\hat{H}_{i}(0), c,-\hat{N}\right)$. Then from the property (3.6), we deduce that such vectors have weight $\xi_{+} \equiv \xi_{\tilde{\omega}}+\tilde{\varphi}$ with $\tilde{\varphi}(\neq 0) \in \widetilde{\Delta}(\mathfrak{h})_{+}$. Now note the following relations for $\xi_{+}$;

$$
\begin{aligned}
\left|\hat{\rho}+\xi_{+}\right|^{2} & =|\tilde{\omega}(\hat{\Lambda}+\hat{\rho})+\tilde{\varphi}|^{2} \\
& =|\hat{\rho}+\hat{\Lambda}|^{2}+2(\tilde{\omega}(\hat{\Lambda}+\hat{\rho}), \tilde{\varphi})+|\tilde{\varphi}|^{2} .
\end{aligned}
$$

Since $\omega^{-1}\left(\tilde{\Delta}\left(\mathfrak{h}_{+}\right)\right) \in \tilde{\Delta}_{+}$for $\tilde{\omega} \in \tilde{W}^{1}$, the second term of the above equation is non-negative. Therefore we have $|\hat{\rho}+\hat{\Lambda}|<\left|\hat{\rho}+\xi_{+}\right|$, which means that $\xi_{+} \notin D_{\hat{\Lambda}}$ owing to the property i). This proves the assertion.

From the above proposition (and Proposition 3.2), we can conclude that the harmonic cocycle $v \in B_{\hat{\Lambda}, \hat{\xi}}$ representing the irreducible $\hat{\mathfrak{h}}$-module $H^{*}\left(\mathrm{~m}_{+} \otimes \mathbf{C}\left[t, t^{-1}\right], L(\hat{\Lambda})\right)_{\hat{\xi}}$ are in one to one correspondence with the subset $\tilde{W}^{1} \in \tilde{W}$. Explicitly, the correspondence of $\tilde{W}^{1}$ to the highest weights of the irreducible 
$\hat{\mathfrak{h}}$-modules representing the harmonic cocycles is given by the following mapping of $\tilde{\sigma} \in \tilde{W}^{1}$ to $\hat{\xi}_{\tilde{\sigma}} \in \hat{P}(\mathfrak{h})_{+, \mathbf{k}}$ :

$$
\tilde{\sigma} \mapsto \tilde{\xi}_{\tilde{\sigma}, i} \equiv \bar{\xi}_{\tilde{\sigma}, i}+k_{i} \Lambda_{0, i} \quad(0 \leqq i \leqq N),
$$

where $\bar{\xi}_{\tilde{\sigma}}$ means the classical part of $\xi_{\tilde{\sigma}}$ and the subscript $i$ means the projection to each component $P\left(\mathfrak{h}_{i}\right)_{+}$.

Due to the multiplicity one property iv), we can express uniquely the harmonic cocycle $\left|\hat{\Lambda}, \xi_{\tilde{\sigma}}\right\rangle$ for each $\tilde{\sigma} \in \tilde{W}^{1}$ as an element of $B_{\hat{\Lambda}, \hat{\xi}_{\tilde{\sigma}}}$;

$$
\left|\hat{\Lambda}, \xi_{\tilde{\sigma}}\right\rangle=\prod_{\substack{\tilde{\phi} \in \boldsymbol{\Phi}_{\tilde{\sigma}} \\ \tilde{\phi}=\varphi+r \delta}} \psi^{\varphi}(-r)|0\rangle \otimes \mathscr{V}_{\tilde{\sigma}}
$$

with $\mathscr{V}_{\tilde{\sigma}} \in L_{\tilde{\sigma}(\hat{\Lambda})}(\hat{\Lambda}) \equiv\{v \in L(\hat{\Lambda}) \mid H(0) v=\langle H, \tilde{\sigma}(\hat{\Lambda})\rangle v\}$. The degree of the harmonic cocycle is easily determined from (3.26) by evaluating the eigenvalue for the fermion number operator $J_{f}(0)$.

Summarizing our results above, we obtain;

\section{Theorem 3.8.}

i) The mapping $\tilde{\sigma} \mapsto H^{*}\left(\mathrm{~m}_{+} \otimes \mathbf{C}\left[t, t^{-1}\right], L(\hat{\Lambda})\right)_{\hat{\xi}_{\tilde{\sigma}}}$, is a bijection of $\tilde{W}^{1}$ onto the set of all irreducible components of $H^{*}\left(\mathfrak{m}_{+} \otimes \mathbf{C}\left[t, t^{-1}\right], L(\hat{\Lambda})\right)$ as the $\hat{\mathfrak{h}}-$-module. And the multiplicity of $H^{*}\left(\mathfrak{m}_{+} \otimes \mathbf{C}\left[t, t^{-1}\right], L(\hat{\Lambda})\right)_{\hat{\xi}}$ is one.

ii) The highest weight vector (3.26) has a meaning as a representative harmonic cocycle in $H^{*}\left(\mathrm{~m}_{+} \otimes \mathbf{C}\left[t, t^{-1}\right], \underline{L}(\hat{\Lambda})\right)_{\hat{\xi}_{\tilde{\sigma}}}$.

iii) The degree of the cocycle $\left|\tilde{\Lambda}, \xi_{\tilde{\sigma}}\right\rangle$ is given by the fermion number $\tilde{l}(\tilde{\sigma})$;

$$
\tilde{l}(\tilde{\sigma}) \equiv \#\left\{\varphi \in \tilde{\Delta}_{+}^{(+)} \mid \varphi \in \Phi_{\tilde{\sigma}}\right\}-\#\left\{\varphi \in \tilde{\Delta}_{+}^{(-)} \mid \varphi \in \Phi_{\tilde{\sigma}}\right\},
$$

with

$$
\tilde{\Delta}_{+}^{( \pm)} \equiv\left\{\gamma \in \tilde{\Delta}_{+} \mid \gamma= \pm \beta+m \delta, \beta \in \Delta_{+}, m \in \mathbf{Z}\right\}
$$

Remark. The harmonic cocycle for NS-type SCA can be obtained from (3.26) by simply shifting the argument of the fermion operator: $\psi^{\varphi}(-r)$ to $\psi^{\varphi}\left(-r \mp \frac{1}{2}\right)$ for $\varphi \in \Delta\left(m_{ \pm}\right)$, respectively. This fact follows from the isomorphisms (4.7) which we will formulate in the next section. Thus there is no essential difference between the two.

Theorem 3.8 is the analogue of the Theorem 5.14 in ref. [Kos] obtained for the classical Lie algebra pairs $g$ and $\mathfrak{h}$. The extensions of Kostant's theory to the case of $\hat{g}$ and a classical $\mathfrak{h}$ have been done in refs. [GL, Kum]. In this context, our arguments are for pairs of the affine Lie algebras $\hat{\mathrm{g}}$ and $\hat{\mathfrak{h}}$. Though our arguments for the (affine) Lie algebra cohomology theory for the pair $\hat{\mathrm{g}}$ and $\hat{\mathfrak{h}}$ is quite parallel to Kostant's theory, we realize, in the latter half of his section, that a characteristic feature appears in determining the subset $\widetilde{W}^{1} \cong \widetilde{W}$ for our case of the affine Lie algebras.

The modified length function $\tilde{l}(\tilde{\omega})$ for $\tilde{W}$, which we have naturally introduced in iii) above as the fermion number, has been introduced by Feigin and Frenkel [FF1, FF2] in their recent work on the BGG (Bernstein-Gelfand-Gelfand) resolution [BGG] for the affine Lie algebra. We present here the explicit expression of the function $\tilde{l}$ for the element $\tilde{\omega}=t_{\gamma} \omega \in \tilde{W}$ :

$$
\tilde{l}\left(t_{\gamma} \omega\right)=l(\omega)-2(\rho, \gamma)
$$


This formula should be constructed to the usual length function $l$;

$$
\left.l\left(t_{\gamma} \omega\right)=\sum_{\alpha \in \Delta_{+} \cap \Delta_{+}}|(\alpha, \gamma)|+\sum_{\beta \in \omega \Delta_{-} \cap \Delta_{+}} \mid 1-(\beta, \gamma)\right) \mid .
$$

The results by Kostant for the classical Lie algebras can be recovered by taking the "classical limit", i.e. $G^{+}(0) \rightarrow Q, G^{-}(0) \rightarrow Q^{*}$ and $\tilde{l}(\tilde{\sigma}) \rightarrow l(\sigma)$ with

$$
Q=\sum_{\alpha \in \Delta\left(\mathfrak{m}_{+}\right)} \psi^{\alpha} J_{\alpha}-\frac{1}{2} \sum_{\alpha, \beta, \gamma \in \Delta\left(\mathfrak{m}_{+}\right)} f_{\alpha \beta}{ }^{\gamma} \psi^{\alpha} \psi^{\beta} \psi_{\gamma},
$$

and $Q^{*}=Q^{\dagger}$. The operators $Q$ and $Q^{*}$ give the Fock space representation for the cohomology operators defined, in the ref. [Kos], on $\wedge m_{+}^{*} \otimes V^{\lambda}$ where $V^{\lambda}$ is a $\mathrm{g}$-module with the highest weight $\lambda$. It is a simple exercise for readers to calculate the Laplacian for the operator $Q$.

(3-3) The Subset $\tilde{W}^{1}$. In the remaining part of this section, we give a characterization of the subset $\tilde{W}^{1}$ of the affine Weyl group $\tilde{W}$.

Let us first note that the following three conditions for the element $\tilde{\omega} \in \tilde{W}^{1}$ are equivalent;

$$
\begin{array}{ll}
\text { 1) } & \tilde{\omega}\left(\tilde{\Delta}_{-}\right) \cap \tilde{\Delta}_{+} \leqq \tilde{\Delta}(\mathfrak{m})_{+}, \\
\text {2) } & \tilde{\omega}^{-1}\left(\tilde{\Delta}(\mathfrak{h})_{+}\right) \subseteq \tilde{\Delta}_{+}, \\
\text {3) } & \tilde{\omega}\left(\tilde{\Delta}_{-}\right) \cap \tilde{\Delta}(\mathfrak{h})_{+}=\phi .
\end{array}
$$

Now define a subgroup $\tilde{\mathfrak{D}} \in \tilde{W}$ by

$$
\widetilde{\mathfrak{D}} \equiv\left\{\tilde{\sigma}=t_{\gamma} \sigma \in \tilde{W} \mid \tilde{\sigma}\left(\tilde{\Delta}(\mathfrak{h})_{+}\right)=\tilde{\Delta}(\mathfrak{h})_{+}, \sigma \in W_{1}\right\},
$$

where $W_{1}$ represents the Weyl group of $\mathfrak{h}$. Then we can easily see from (3.33) that the subset $\widetilde{W}^{1}$ admits the action of $\tilde{\mathfrak{D}}$, i.e.,

$$
\tilde{\mathfrak{D}} \cdot \tilde{W}^{1}=\tilde{W}^{1} .
$$

Here our main assertion may be stated as;

Proposition 3.9. The subset $\tilde{W}^{1}$ decomposes uniquely as

$$
\tilde{W}^{1}=\tilde{\mathfrak{D}} \cdot W^{1},
$$

where $W^{\prime}$ represents the classical counterpart of $\tilde{W}^{\prime}$.

To prove this assertion, we first note the conditions for $\tilde{\omega}=t_{\gamma} \omega \in \tilde{W}$ to belong to the $\widetilde{W}^{1}$ can be written as

a) $(\gamma, \varphi)=0$ or 1 for arbitrary $\varphi \in \Delta(\mathfrak{h})_{+}$

b) $\omega^{-1}(\varphi) \in \Delta_{+}$for $\varphi \in \Delta(\mathfrak{h})_{+}$such that $(\gamma, \varphi)=0$,

c) $\omega^{-1}(\varphi) \in \Delta_{-}$for $\varphi \in \Delta(\mathfrak{h})_{+}$such that $(\gamma, \varphi)=1$.

Similarly for $\tilde{\sigma}=t_{\gamma^{\prime}} \sigma \in \tilde{W}$ to belong to $\widetilde{D}$ we must have

a) $\left(\gamma^{\prime}, \varphi\right)=0$ or 1 for arbitrary $\varphi \in \Delta(\mathfrak{h})_{+}$

b) $\sigma^{\prime} \sigma^{-1}(\varphi) \in \Delta(\mathfrak{h})_{+}$for $\varphi \in \Delta(\mathfrak{h})_{+}$such that $\left(\gamma^{\prime}, \varphi\right)=0$,

c) ${ }^{\prime} \sigma^{-1}(\varphi) \in \Delta(\mathfrak{h})$ - for $\varphi \in \Delta(\mathfrak{h})_{+}$such that $\left(\gamma^{\prime}, \varphi\right)=1$.

We can see the existence of the elements $\tilde{\sigma} \in \widetilde{D}$ satisfying a) $\left.)^{\prime}-c\right)^{\prime}$, by noting that;

Lemma 3.10. For any elements $\gamma^{\prime} \in M$ satisfying the condition a) above, there exists a unique element $\sigma \in W_{1}$ as a solution for $\left.\mathrm{b}\right)^{\prime}$ and c)'.

Proof. Define $\Delta(\mathfrak{h})_{+}^{0} \equiv\left\{\varphi \in \Delta(\mathfrak{h})_{+} \mid\left(\gamma^{\prime}, \varphi\right)=0\right\}$ and $\Delta(\mathfrak{h})_{+}^{1} \equiv\left\{\varphi \in \Delta(\mathfrak{h})_{+} \mid\left(\gamma^{\prime}, \varphi\right)=1\right\}$. Then 
we see that both $\Delta(\mathfrak{h})_{+}^{0}$ and $\Delta(\mathfrak{h})_{+}^{1}$ are closed under the dot addition $\dot{+}$ defined in $\Delta$ by addition in case the sum again lies in $\Delta$ and otherwise zero operation. Let $\Delta(\mathfrak{h})_{\gamma^{\prime}} \equiv \Delta(\mathfrak{h})_{+}^{0} \sqcup\left(-\Delta(\mathfrak{h})_{+}^{1}\right)$, then the conditions b) $)^{\prime}$ and c c) can be summarized as

$$
\sigma^{-1}\left(\Delta(\mathfrak{h})_{\gamma^{\prime}}\right)=\Delta(\mathfrak{h})_{+} \cdot
$$

Since $\Delta(\mathfrak{h})_{\gamma^{\prime}}$ is closed under $\dot{+}$ operation and $\Delta(\mathfrak{h})=\Delta(\mathfrak{h})_{\gamma^{\prime}} \sqcup\left(-\Delta(\mathfrak{h})_{\gamma^{\prime}}\right.$, there exists a unique element $\sigma \in W_{1}$ satisfying (3.37), (see e.g. ref. [Kos], Proposition 5.10).

Noting the equality of the conditions a) for $\tilde{W}^{1}$ and a)' for $\tilde{\mathfrak{D}}$, we can conclude that for arbitrary $\tilde{\omega}=t_{\gamma} \omega \in \tilde{W}^{1}$ there exist a unique element $\tilde{\sigma}=\tau_{\gamma} \sigma \in \tilde{D}$ and then

$$
\tilde{\sigma}^{-1} \tilde{\omega}=\sigma^{-1} \omega \in W^{1} .
$$

This completes our proof for Proposition 3.9.

Now we study the lattice elements $\gamma \in M$ which satisfies the condition a). To this aim, we define the coroot lattice of $\mathfrak{h}$ by $Q(\mathfrak{h})^{\vee} \equiv Q_{0} \oplus Q_{1}^{\vee} \oplus \cdots \oplus Q_{N}^{\vee} \subseteq Q^{\vee}(=M)$, including the lattice $Q_{0}=Q^{\vee} \cap t_{0}^{*}$ for $\mathfrak{h}_{0}$, with $Q_{i}^{\vee} \equiv \sum_{s=1}^{l_{i}} \mathbf{z}_{\frac{2}{\left(\alpha_{(s) i}, \alpha_{(s) i}\right)}} \alpha_{(s) i}(1 \leqq i \leqq N)$. It is convenient to use the notation of $Q^{\vee}$ rather than $M$ in the following arguments.

Proposition 3.11. There is a bijection between the set of the lattice elements $\gamma \in Q^{\vee}$ $\bmod Q_{0}$ satisfying the condition a) and the $\operatorname{coset} Q^{\vee} / Q(\mathfrak{h})^{\vee}$.

To show this property we prepare the following lemma;

\section{Lemma 3.12.}

1) We can take a representative element $\gamma_{1}$ of the class $[\gamma] \in Q^{\vee} / Q(\mathfrak{h})^{\vee}$ so that its length $\left|\gamma_{1}\right|$ is minimum and $\left(\gamma_{1}, \varphi\right) \geqq 0$ for $\forall \varphi \in \Delta(\mathfrak{h})_{+}$.

2) Such $\gamma_{1} \in[\gamma]$ is determined uniquely $\bmod Q_{0}$ and satisfies $\left(\gamma_{1}, \varphi\right)=0$ or 1 for $\forall \varphi \in \Delta(\mathfrak{h})_{+}$.

\section{Proof.}

1) Among the elements $\gamma_{1}$ 's $\in[\gamma]$ with minimum length $\left|\gamma_{1}\right|$, we are allowed to consider the action of the Weyl group $W_{1}$, since $\left|\gamma_{1}\right|=\left|r_{\varphi}\left(\gamma_{1}\right)\right|$ and $r_{\varphi}\left(\gamma_{1}\right)=\gamma_{1}-$ $\left(\gamma_{1}, \varphi^{\vee}\right) \varphi=\gamma_{1}-\left(\gamma_{1}, \varphi\right) \varphi^{\vee} \in[\gamma]$ for $\forall r_{\varphi} \in W_{1}$. Therefore we can make any element $\gamma_{1} \in[\gamma]$, which gives the minimum of $\left|\gamma_{1}\right|$, to satisfy $\left(\gamma_{1}, \varphi\right) \geqq 0$ for $\forall \varphi \in \Delta(\mathfrak{h})_{+}$through the action of $W_{1}$.

2) Assume there exists $\varphi \in \Delta(\mathfrak{h})_{+}$such that $\left(\gamma_{1}, \varphi\right) \geqq 2$, then we have

$$
\begin{aligned}
\left|\gamma_{1}-\varphi^{\vee}\right|^{2} & =\left|\gamma_{1}\right|^{2}+\left(\varphi^{\vee}, \varphi^{\vee}-2 \gamma_{1}\right) \\
& =\left|\gamma_{1}\right|^{2}+\frac{4}{(\varphi, \varphi)}\left(1-\left(\varphi, \gamma_{1}\right)\right) .
\end{aligned}
$$

This implies $\left|\gamma_{1}-\varphi^{\vee}\right|<\left|\gamma_{1}\right|$ for $\gamma_{1}-\varphi^{\vee} \in[\gamma]$ and a contradiction. As for the uniqueness, assume that we have obtained two representatives $\gamma_{1}, \gamma_{2} \in[\gamma]$ through the argument 1) above. Then we have $\gamma_{1}-\gamma_{2} \in Q(\mathfrak{h})^{\vee}$ and $\left(\gamma_{1}-\gamma_{2}, \varphi\right)=0$ or \pm 1 for $\forall \varphi \in \Delta(\mathfrak{h})$. Taking an element $\omega_{1} \in W_{1}$ so that $\left(\omega_{1}\left(\gamma_{1}-\gamma_{2}\right), \varphi\right) \geqq 0$ for $\forall \varphi \in \Delta(\mathfrak{h})_{+}$, we can express the above conditions as $\left(\omega_{1}\left(\gamma_{1}-\gamma_{2}\right), \varphi\right)=0$ or 1 for $\forall \varphi \in \Delta(\mathfrak{h})_{+}$with $\omega_{1}\left(\gamma_{1}-\gamma_{2}\right) \in Q(\mathfrak{h})^{\vee}$. Now recall that the closure of the fundamental cell for each $\mathfrak{h}_{i}(1 \leqq i \leqq N)$ is defined by $\bar{D}_{(i)}^{0}=\left\{x \in t_{i \mathbf{R}}^{*} \mid 0 \leqq(\alpha, x) \leqq 1\right.$ for any $\left.\alpha \in \Delta\left(\mathfrak{h}_{i}\right)_{+}\right\}$. Then it is known that $\bar{D}_{(i)}^{(0)} \cap Q\left(\mathfrak{h}_{i}\right)^{\vee}=\{0\}$ (see Corollary 1.20 in ref. [IM]). From this fact we must have $\omega_{1}\left(\gamma_{1}-\gamma_{2}\right) \in Q_{0}$, i.e., $\gamma_{1}=\gamma_{2} \bmod Q_{0}$. 
In this way we can find a lattice element $\gamma \in Q^{\vee}$ mod $Q_{0}$ satisfiying the condition a) in each class $[\gamma] \in Q^{\vee} / Q(\mathfrak{h})^{\vee}$. On the other hand, we realize that there exists only one such element $\gamma \in Q^{\vee} \bmod Q_{0}$ for each class of $Q^{\vee} / Q(\mathfrak{h})^{\vee}$ applying the same argument for the uniqueness of $\gamma_{1}$ in 2) of the above lemma. Thus we complete the proof of Proposition 3.11.

We define the quotient group $\mathfrak{D}$ by

$$
\mathfrak{D} \equiv T_{0} \backslash \tilde{\mathfrak{D}},
$$

with respect to the normal subgroup $T_{0}=\left\{t_{\lambda} \mid(\lambda, \varphi)=0, \varphi \in \Delta(\mathfrak{h})_{+}, \lambda \in M\right\}$. In following sections, it will become clear that the group $\mathfrak{D}$ can be constructed via the diagram automorphisms of the extended Dynkin diagrams for each $\mathfrak{h}_{i}$ 's $(i=1 \cdots N)$.

Remark. In practical calculations, the following characterization of $\gamma \in M$ satisfying the condition a) is useful. To describe this, let us define the lattice $Q_{i}^{\perp} \equiv \sum_{s=1}^{l_{i}} \mathbf{Z} \frac{2 \Lambda_{(s) i}}{\left(\alpha_{(s) i}, \alpha_{(s) i}\right)}=\sum_{s=1}^{l_{i}} \mathbf{Z} e_{(s) i}$ with the fundamental weights $\Lambda_{(s) i}\left(1 \leqq s \leqq l_{i}\right)$ with respect to the simple system $\Pi_{i}$. Then we can easily prove the following:

The set $\Gamma_{i} \equiv\left\{\gamma \in Q_{i}^{\perp} \mid(\gamma, \varphi)=0\right.$ or 1 for $\left.\forall \varphi \in \Delta\left(\mathfrak{h}_{i}\right)_{+}\right\}(1 \leqq i \leqq N)$

consists of 0 and the basis vector $e_{(s) i}$ with $m_{(s) i}=1$ in the expression

$\theta_{i}=\sum_{t=1}^{l_{i}} m_{(t) i} \alpha_{(t) i}$.

Now note that the inclusion relation $M \subset Q_{0}^{\perp} \oplus \sum_{i=1}^{N} Q_{i}^{\perp}$. Then we convince ourselves that we must find $\left(\bmod Q_{0}\right)$ the solutions $\gamma \in M$ for the condition a) in the set $\Gamma \equiv\left\{v_{0}+v_{1}+\cdots+v_{N} \mid v_{0} \in Q_{0}^{\perp}, v_{i} \in \Gamma_{i}(1 \leqq i \leqq N)\right\}$.

\section{Algebra Automorphisms of the $N=2 \mathrm{SCA}$}

Owing to the orthogonal decomposition formulated in Sect. 2 and the complete reducibility for the integrable modules, we have the following isomorphism;

$$
\mathscr{F}_{\varepsilon} \otimes L(\hat{\Lambda}) \cong \sum_{\hat{\xi} \in \hat{P}(\hat{b})_{+, k}} B_{\hat{\Lambda}, \hat{\xi}}^{(\xi)} \otimes L_{\mathfrak{h}}(\hat{\xi}) .
$$

The purpose of this section is to construct the algebra automorphisms for the $N=2$ SCA and utilize them to reveal the structures of the multiplicities of $N=2$ SCA-module which occur in the branching coefficients, $B_{\hat{\lambda}, \xi}^{(\varepsilon)}$. This problem of the multiplicities is known, by physicist [Gep], as the field identification problem. We first approach this by formulating an automorphism known as the spectral flow. Later we relate our results to the modular transformation property of the GKO branching coefficients.

Other automorphisms we will formulate are the spectral flow which connect $\mathscr{F}_{0}$ to $\mathscr{F}_{1 / 2}$ and the charge conjugation automorphism. The former automorphism completes our calculations of the cohomology group which we have done only for the R-type SCA in the previous section. The latter automorphism will prove to be useful in revealing a certain duality property of the Poincare polynomials which we will define in Sect. 5. 
(4-1) Spectral Flow. To formulate the automorphism, we first supplement the properties of the affine Weyl group to those given in Sect. 3.

Consider the affine Weyl group $\tilde{W} \subset G L\left(\tilde{\mathfrak{t}}^{*}\right)$ and $\tilde{W}_{1} \equiv \tilde{W}\left(\mathfrak{h}_{1}\right) \times \cdots \times \tilde{W}\left(\mathfrak{h}_{N}\right) \subset$ $G L\left(\tilde{\mathfrak{t}}_{1}^{*} \oplus \cdots \oplus \tilde{\mathfrak{t}}_{N}^{*}\right)$ for $\tilde{\mathfrak{g}}$ and $\tilde{\mathfrak{h}}$, respectively. Precisely the affine Weyl group $\tilde{W}\left(\mathfrak{h}_{i}\right)(1 \leqq i \leqq N)$ is defined by the simple reflections $r_{j}^{(i)}$ with respect to $\alpha_{(j) i} \in \tilde{\Pi}_{i}$ defined with the normalized Cartan-Killing form $(,)_{i}$ and is expressed as $T_{(i)} \ltimes W\left(\mathfrak{h}_{i}\right)$. Where $T_{(i)}(1 \leqq i \leqq N)$ represents the translation group associated with the long root lattice $M_{i}$ of each $\mathfrak{h}_{i}$ and $t_{\gamma}^{(i)}$ acts on $\lambda \in \tilde{\mathrm{t}}_{i}^{*}$ as

$$
t_{\gamma}^{(i)}(\lambda)=\lambda+\left(\lambda, \delta_{i}\right)_{i} \gamma_{i}-\left[(\lambda, \gamma)_{i}+\frac{1}{2}\left(\lambda, \delta_{i}\right)_{i}(\gamma, \gamma)_{i}\right] \delta_{i}
$$

When we regard $\tilde{\mathrm{t}}_{1}^{*} \oplus \cdots \oplus \tilde{\mathrm{t}}_{N}^{*}$ as the subspace of $\tilde{\mathrm{t}}^{*}$ under the inclusion $\mathrm{t}_{1}^{*} \oplus \cdots$ $\oplus \mathrm{t}_{N}^{*} \subseteq \mathrm{t}^{*}$ and the identification $v:\left(\Lambda_{(0) i}, \delta_{i}\right) \rightarrow\left(\frac{\left(\theta_{i}, \theta_{i}\right)}{2} \Lambda_{(0)}, \delta\right)$, we have a relation $t_{\left.\left(\theta_{i}, \theta_{i}\right) / 2\right) \gamma}^{(\lambda)}(\lambda)=t_{\gamma}(v(\lambda))$ and, thus, the affine Weyl group $\tilde{W}\left(\mathfrak{h}_{i}\right)$ can be identified, as a subgroup of $\tilde{W}$, with $T_{i} \ltimes W\left(\mathfrak{h}_{i}\right)$, where $T_{i} \equiv\left\{t_{\gamma} \in T \mid \gamma \in Q_{i}^{\vee} \subseteq Q^{\vee}\right\}$. It is convenient to use this identification of $\tilde{W}\left(\mathfrak{h}_{i}\right)(1 \leqq i \leqq N)$ when we regard the $\tilde{W}\left(\mathfrak{h}_{i}\right)$ as a subgroup of $\widetilde{W}$.

Next let us consider the action of the Weyl group $\tilde{W}$ on an integrable $\hat{\mathrm{g}}$-module $(L(\hat{\Lambda}), \pi)$. It is known (see ref. [Kac] Sect. 12.8) that the affine Weyl group $\tilde{W}$ has a (projective) realization $\tilde{W}^{\pi}$ on the module $L(\tilde{\Lambda})$ by the correspondence; $r_{i_{1}} \cdots r_{i_{n}} \in \tilde{W} \mapsto r_{i_{1}}^{\pi} \cdots r_{i_{n}}^{\pi} \in \tilde{W}^{\pi}$ with

$$
\begin{aligned}
& r_{i}^{\pi}=\exp \left(J_{-\alpha_{(i)}}^{\vee}(0)\right) \exp \left(-J_{\alpha_{(i)}}^{\vee}(0)\right) \exp \left(J_{-\alpha_{(i)}}^{\vee}(0)\right), \\
& r_{0}^{\pi}=\exp \left(J_{\theta}(-1)\right) \exp \left(-J_{-\theta}(1)\right) \exp \left(J_{\theta}(-1)\right),
\end{aligned}
$$

where $J_{\alpha}^{\vee}(n) \equiv \sqrt{\frac{2}{(\alpha, \alpha)}} J_{\alpha}(n)$. This means that, for $i=1, \ldots, l$, we have $r_{i}^{\pi} J_{\alpha}(n)\left(r_{i}^{\pi}\right)^{-1}=$ $J_{r_{i}(\alpha)}(n)$ and, for $t_{\gamma} \in T$,

$$
\begin{aligned}
t_{\gamma}^{\pi} J_{\alpha}(n)\left(t_{\gamma}^{\pi}\right)^{-1} & =J_{\alpha}(n-(\alpha, \gamma)), \\
t_{\gamma}^{\pi} H_{\alpha}(n)\left(t_{\gamma}^{\pi}\right)^{-1} & =H_{\alpha}(n)-c\left\langle\gamma, H_{\alpha}\right\rangle \delta_{n, 0}, \\
t_{\gamma}^{\pi} T(n)\left(t_{\gamma}^{\pi}\right)^{-1} & =T(n)-H_{\gamma}(n)-\frac{c}{2}(\gamma, \gamma) \delta_{n, 0} .
\end{aligned}
$$

Therefore if $L(\hat{\Lambda})=\sum_{\lambda \in P(\hat{\Lambda})} L_{\lambda}(\hat{\Lambda})$ is the weight space decomposition, then $\omega^{\pi} \in \tilde{W}^{\pi}$ acts as $\omega^{\pi}\left(L_{\lambda}(\hat{\Lambda})\right)=L_{\omega(\lambda)}(\hat{\Lambda})$. In this way, the affine Weyl group acts on the module $L(\hat{\Lambda})$.

In the following arguments, we will use several lattice in $\mathrm{t}^{*}$. We define the dual lattices $Q^{\perp}$ and $P^{\perp}(=M)$ for $Q$ and $P$, respectively, by

$$
\begin{aligned}
& Q^{\perp}=\left\{\lambda \in \mathfrak{t}^{*} \mid(\lambda, \alpha) \in \mathbf{Z}, \alpha \in Q\right\}, \\
& P^{\perp}=\left\{\lambda \in \mathfrak{t}^{*} \mid(\lambda, \Lambda) \in \mathbf{Z}, \Lambda \in P\right\} .
\end{aligned}
$$

Recall that the root lattices of $\mathfrak{h}_{i}(1 \leqq i \leqq N)$ and $Q_{0}$ are defined as the sublattices of $Q$. Then each root lattice $Q_{i}(0 \leqq i \leqq N)$ is naturally endowed with the induced form previously defined in Sect. 2 . With this inner product on $Q_{i}(0 \leqq i \leqq N)$, we define the dual lattices $Q_{i}^{\perp}$;

$$
Q^{\perp} \subseteq Q_{0}^{\perp} \oplus Q_{1}^{\perp} \oplus \cdots \oplus Q_{N}^{\perp} .
$$


Now let us extend the affine Weyl group $\tilde{W}$ of $\tilde{\mathfrak{g}}$ to a slightly larger group $\tilde{W}_{0} \equiv T^{\perp} \ltimes W$ with $T^{\perp} \equiv\left\{t_{\lambda} \mid \lambda \in Q^{\perp}\right\}$ following Kac-Wakimoto [KW]. Then it is known that this extended group $\tilde{W}_{0}$ splits into the semi-direct product as

$$
\tilde{W}_{0}=\tilde{W}_{0}^{+} \cdot \tilde{W},
$$

where the subgroup $\tilde{W}_{0}^{+} \subseteq \tilde{W}_{0}$ is defined by

$$
\tilde{W}_{0}^{+} \equiv\left\{\tilde{\omega} \in \tilde{W}_{0} \mid \tilde{\omega}\left(\tilde{\Delta}_{+}\right)=\tilde{\Delta}_{+}\right\} .
$$

This property (4.10) can be deduced as follows: We easily verify that the extended group acts on the set of all cells in $\mathfrak{t}_{\mathbf{R}}^{*}$. On the other hand, we know that the affine Weyl group $\tilde{W}$ acts simply and transitively on the set of all cells. Therefore we can find for arbitrary $\tilde{\omega} \in \tilde{W}_{0}$ a unique element $\tilde{\sigma} \in \tilde{W}$ so that the product $\tilde{\omega} \tilde{\sigma}^{-1} \equiv \tilde{\rho}$ acts trivially on the cells, i.e., $\tilde{\rho} \in \tilde{W}_{0}^{+}$.

The following commutative diagram indicates that there is an isomorphism of the groups between $W_{0}^{+}$and $Q^{\perp} / M(\cong$ center of $G)$ :

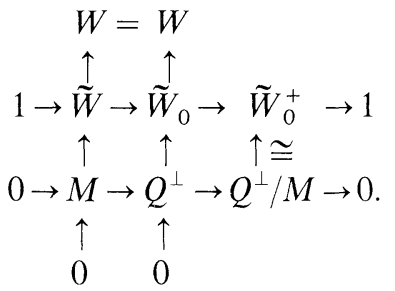

In the following, we study the extension of the action $\tilde{W}^{\pi}$ of the affine Weyl group on $g$-module to that of the extended group $\tilde{W}_{0}$. Then the sum of $\hat{\mathrm{g}}$-modules $L_{k} \equiv \sum_{\hat{\Lambda} \in P_{+, k}} L(\hat{\Lambda})$ naturally arises.

Proposition 4.1. (Spectral flow I). Define following automorphisms of the Clifford algebra and the affine Lie algebra $\tilde{\mathfrak{g}}$ for each $\gamma \in Q^{\perp}$;

i) for the Clifford algebra, let

$$
\sigma_{\gamma}^{f}\left(\psi^{\alpha}(r)\right)=\psi^{\alpha}(r+(\gamma, \alpha)), \quad \alpha \in \Delta\left(\mathrm{m}_{+}\right) \sqcup \Delta\left(\mathrm{m}_{-}\right) .
$$

ii) For $\tilde{\mathfrak{g}}$ let

$$
\begin{aligned}
t_{\gamma}^{\mathrm{ad}}\left(J_{\alpha}(n)\right) & =J_{\alpha}(n-(\gamma, \alpha)), \quad(\alpha \in \Delta) \\
t_{\gamma}^{\mathrm{ad}}\left(H_{\alpha}(n)\right) & =H_{\alpha}(n)-c\left\langle\gamma, H_{\alpha}\right\rangle \delta_{n, 0}, \\
t_{\gamma}^{\mathrm{ad}}(d) & =d+H_{\gamma}(0)+\frac{c}{2}(\gamma, \gamma), \quad t_{\gamma}^{\mathrm{ad}}(c)=c,
\end{aligned}
$$

with $\gamma \in Q^{\perp}$ and $d \equiv-T_{\mathrm{g}}(0)$.

Then the actions $\sigma_{\gamma}$ and $t_{\gamma}^{\text {ad }}$ are implementable on the modules $\mathscr{F}_{\varepsilon}$ and $L_{k}$, that is, there exists a linear isomorphism $\tilde{\sigma}_{\gamma}^{f}: \mathscr{F}_{\varepsilon} \rightarrow \mathscr{F}_{\varepsilon}$ and $\tilde{t}_{\gamma}: L_{k} \rightarrow L_{k}$ s.t.

$$
\begin{aligned}
\tilde{\sigma}_{\gamma}^{f} \psi^{\alpha}(r)\left(\tilde{\sigma}_{\gamma}^{f}\right)^{-1} & =\sigma_{\gamma}^{f}\left(\psi^{\alpha}(r)\right), \\
\tilde{t}_{\gamma} X\left(\tilde{t}_{\gamma}\right)^{-1} & =t_{\gamma}^{\mathrm{ad}}(X), \quad X \in \tilde{\mathfrak{g}} .
\end{aligned}
$$

These actions $\tilde{\sigma}_{\gamma}^{f}$ and $\tilde{t}_{\gamma}$ define the projective representations of $\gamma \in Q^{\perp}$. 
We do not go into the detailed proof but only explain how to construct the linear isomorphisms $\tilde{\sigma}_{\gamma}^{f}$ and $\tilde{t}_{\gamma}$.

Table 1. Diagram automorphisms $\tilde{W}_{0}^{+}$

\begin{tabular}{lll}
\hline & \multicolumn{2}{c}{$\tilde{W}_{0}^{+}$} \\
\hline$A_{l}$ & $1, \tilde{\rho}, \ldots, \tilde{\rho}^{l}$ & $; \tilde{\rho}=t_{\varepsilon_{1}} \omega_{\Pi_{1}} \omega_{\Pi}$ \\
$B_{l}$ & $1, \tilde{\rho}$ & $; \tilde{\rho}=t_{\varepsilon_{1}} \omega_{\Pi_{1}} \omega_{\Pi}$ \\
$C_{l}$ & $1, \tilde{\rho}$ & $; \tilde{\rho}=t_{\varepsilon_{1}} \omega_{\Pi_{l}} \omega_{\Pi}$ \\
$D_{l}$ & $1, \tilde{\rho}_{1}, \tilde{\rho}_{l-1}, \tilde{\rho}_{l}$ & $; \tilde{\rho}_{i}=t_{\varepsilon_{i}} \omega_{\Pi_{i}} \omega_{\Pi_{i}} \omega_{\Pi \Pi}(i=1, l-1, l)$ \\
$E_{6}$ & $1, \tilde{\rho}, \tilde{\rho}^{2}$ & $; \tilde{\rho}=t_{\varepsilon_{1}} \omega_{\Pi_{1}} \omega_{\Pi}$ \\
$E_{7}$ & $1, \tilde{\rho}$ & $; \tilde{\rho}=t_{\varepsilon_{1}} \omega_{\Pi_{1}} \omega_{\Pi}$ \\
$E_{8}, F_{4}, G_{2}$ & 1 & \\
\hline
\end{tabular}

The elements of the diagram automorphisms $\tilde{W}_{0}^{+}$are listed. $\omega_{\Pi}$ represents the longest element of the Weyl group $W$ and $\omega_{\Pi_{i}}$ is defined by $\omega_{\Pi_{i}}\left(\Pi \backslash\left\{\alpha_{(i)}\right\}\right)=$ $-\left(\left\{\Pi \backslash\left\{\alpha_{(i)}\right\}\right)\right.$. For example the $\tilde{\rho}$ for $A_{l}$ generate the $\mathbf{Z}_{l+1}$ symmetry, $\tilde{\rho} r_{i} \tilde{\rho}^{-1}=r_{i+1 \bmod l+1}$, and in this case $\omega_{\Pi_{1}} \omega_{\Pi}$ coincides with the cyclic permutation.

The former isomorphism $\tilde{\sigma}_{\gamma}^{f}$ is easily constructed on the Fock space as the operation of changing the vacuum vector. As for the latter isomorphism $\tilde{t}_{\gamma}$, let us study the group action of $\tilde{W}_{0}$ on the module $L_{k}$.

Owing to the property (4.10), we can find for arbitrary $\tilde{\omega} \in \tilde{W}_{0}$ a unique element $\tilde{\sigma} \in \tilde{W}$ s.t. $\tilde{\omega} \tilde{\sigma}^{-1}=\tilde{\rho} \in \tilde{W}_{0}^{+}$. Then the element $\tilde{\omega}=t_{\gamma} \omega(=\tilde{\rho} \tilde{\sigma}) \in \tilde{W}_{0}$ defines the algebra automorphism of $\tilde{\mathfrak{g}}$ by $\tilde{\omega}^{\text {ad }}=t_{\gamma}^{\text {ad }} \omega^{\text {ad }}\left(=\tilde{\rho}^{\text {ad }} \tilde{\sigma}^{\text {ad }}\right)$. We know that the action $\tilde{\sigma}^{\text {ad }}$ is implementable on $\hat{\mathfrak{g}}$-module $L(\hat{\Lambda})$ since $\tilde{\sigma} \in \tilde{W}$. As for the action $\tilde{\rho}^{\text {ad }}\left(\tilde{\rho} \in \tilde{W}_{0}^{+}\right)$, on the other hand, we must formulate in a different way: First let us note that $\tilde{\rho}^{\text {ad }} \in$ Aut $\tilde{\mathrm{g}}$ acts on the Cartan subalgebra $\tilde{\mathrm{t}}$ and induces a map $\tilde{\Delta}_{ \pm} \rightarrow \tilde{\Delta}_{ \pm}$. Then it follows that this map induces a permutation $\tilde{\rho}: \alpha_{(i)} \rightarrow \alpha_{(\rho(i))}$ on the set of simple roots $\tilde{\Pi}$. This permutation, in turn, defines a linear map on $\hat{P}_{+}$by $\tilde{\rho}: \hat{\Lambda}_{(i)} \rightarrow \hat{\Lambda}_{(\rho(i))}(0 \leqq i \leqq l)$ with $\hat{\Lambda}_{(i)}$ representing the simple weight. Thus we realize that we can implement the action $\tilde{\rho}^{\text {ad }}$ on the module $L_{k}$ by defining $\tilde{\rho}^{\pi}: J_{1}\left(n_{1}\right) \cdots J_{s}\left(n_{s}\right)|\hat{\Lambda}\rangle \in L(\hat{\Lambda}) \mapsto \rho^{\text {ad }}\left(J_{1}\left(n_{1}\right)\right) \cdots$ $\rho^{\text {ad }}\left(J_{s}\left(n_{s}\right)\right)|\tilde{\rho}(\hat{\Lambda})\rangle \in L(\tilde{\rho}(\hat{\Lambda}))$.

Since the action $\tilde{\rho}: \alpha_{(i)} \rightarrow \alpha_{(\rho(i))}$, preserves the form of the extended Cartan matrix, we deduce $W_{0}^{+} \subset$ Aut $\tilde{\Pi}$. In fact, it is known (Proposition 1.3 in ref. [KW]) that the group $W_{0}^{+}$is the only normal subgroup of Aut $\tilde{\Pi}$ and Aut $\tilde{\Pi}=W_{0}^{+} \ltimes$ Aut $\Pi$. We listed in Table 1 the elements of $\tilde{W}_{0}^{+}$which have been obtained in refs. [IM, Has].

From the above considerations, we see that the action $t_{\gamma}^{\text {ad }}\left(\gamma \in Q^{\perp}\right)$ on $\hat{\mathrm{g}}$ is implemented projectively on the module $L_{k}$ by the successive action of the diagram automorphism $\tilde{\rho}^{\pi}$ and the Weyl group $\tilde{\omega}^{\pi}$, which are determined uniquely so that $t_{\gamma}=\tilde{\omega} \tilde{\rho}_{\gamma}$, in the following way;

$$
\tilde{t}_{\gamma}(|\hat{\Lambda}\rangle)=\tilde{\omega}^{\pi} \tilde{\rho}_{\gamma}^{\pi}|\hat{\Lambda}\rangle
$$

Now we can proceed to our relative case, $(\hat{\mathfrak{g}} \supseteqq \hat{\mathfrak{h}})$;

Proposition 4.2. (Spectral flow II). Consider the diagonal action $\sigma_{\gamma} \equiv \sigma_{\gamma}^{f}+t_{\gamma}^{\text {ad }}$ with $\gamma \in Q^{\perp}$, then 
i) $\sigma_{\gamma}$ acts trivially on the $N=2 \mathrm{SCA}$;

$$
\sigma_{\gamma}(J(n))=J(n), \quad \sigma_{\gamma}\left(G^{ \pm}(r)\right)=G^{ \pm}(r), \quad \sigma_{\gamma}(T(n))=T(n),
$$

ii) $\sigma_{\gamma}$ acts on the $\hat{\mathfrak{h}}_{i}$-currents $\left(1 \leqq i \leqq N, \alpha \in \Delta\left(\mathfrak{h}_{i}\right)\right)$ as

$$
\begin{aligned}
\sigma_{\gamma}\left(\hat{J}_{\alpha}(n)\right) & =\hat{J}_{\alpha}\left(n-\left(\frac{\left(\theta_{i}, \theta_{i}\right)}{2} \gamma_{i}, \alpha\right)_{i}\right), \\
\sigma_{\gamma}\left(\hat{H}_{\alpha}(n)\right) & =\hat{H}_{\alpha}(n)-c_{i}\left\langle\frac{\left(\theta_{i}, \theta_{i}\right)}{2} \gamma_{i}, H_{\alpha}\right\rangle \delta_{n, 0}, \\
\sigma_{\gamma}\left(\hat{d}_{i}\right) & =\hat{d}_{i}+\hat{H}_{\gamma_{i}}(0)+\frac{c_{i}}{2}\left(\frac{\left(\theta_{i}, \theta_{i}\right)}{2} \gamma_{i}, \frac{\left(\theta_{i}, \theta_{i}\right)}{2} \gamma_{i}\right)_{i} \delta_{n, 0}, \quad \sigma_{\gamma}\left(c_{i}\right)=c_{i},
\end{aligned}
$$

and for the abelian part $\mathfrak{h}_{0}$ as

$$
\begin{aligned}
& \sigma_{\gamma}\left(\hat{H}_{a}(n)\right)=\hat{H}_{\alpha}(n)-c_{0}\left(\gamma_{0}, a\right) \delta_{n, 0}, \quad\left(a \in \mathscr{A}_{0}\right), \\
& \sigma_{\gamma}\left(\hat{d}_{0}\right)=\hat{d}_{0}+\hat{H}_{\gamma_{0}}(0)+\frac{c_{0}}{2}\left(\gamma_{0}, \gamma_{0}\right) \delta_{n, 0}, \quad \sigma_{\gamma}\left(c_{0}\right)=c_{0},
\end{aligned}
$$

where $\gamma_{0}, \ldots, \gamma_{l}$ means the projected components of $\gamma$ and $\hat{d}_{i}=-\hat{T}_{\mathrm{h}_{i}}(0), c_{i}=$ $\frac{2}{\left(\theta_{i}, \theta_{i}\right)}\left(c+g^{*}-h_{i}^{*} \frac{\left(\theta_{i}, \theta_{i}\right)}{2}\right)(1 \leqq i \leqq N), c_{0}=c+g^{*}$.

iii) The action $\sigma_{\gamma}$ on $\tilde{\mathfrak{h}}_{i}(0 \leqq i \leqq N)$ defines the algebra automorphism for each $\mathfrak{h}_{i}$ and implementable on the $\hat{\mathfrak{h}}_{i}$-modules $L_{k_{i}} \equiv \sum_{\hat{\xi} \in \hat{P}\left(\mathfrak{h}_{i}\right)_{+, k_{i}}} L_{\mathfrak{h}_{i}}(\widehat{\xi}),(0 \leqq i \leqq N)$ by a linear
isomorphism $\tilde{\sigma}_{\gamma_{i}}(0 \leqq i \leqq N)$.

These automorphisms i), ii) follow from direct evaluations of the action $\rho_{\gamma}$ on each operator. The linear isomorphism $\tilde{\sigma}_{\gamma_{i}}$ can be constructed on $L_{k_{i}}(1 \leqq i \leqq N)$ in the same way as (4.19) for $\tilde{\mathfrak{g}}$;

$$
\sigma_{\gamma_{i}}\left(\left|\hat{\xi}_{i}\right\rangle\right)=\tilde{t}_{\left(\left(\theta_{i}, \theta_{i}\right) / 2\right) \gamma_{i}}^{(i)}\left(\left|\widehat{\xi}_{i}\right\rangle\right)
$$

where $\tilde{t}_{\left(\left(\theta_{i}, \theta_{i}\right) / 2\right) \gamma_{i}}^{(i)}$ belongs to the extended group $T_{(i)}^{\perp} \equiv\left\{t_{\gamma}^{(i)} \mid \gamma \in Q_{(i)}^{\perp}\right\}$ of $T_{(i)}$ with the definition $Q_{(i)}^{\perp} \equiv\left\{\lambda \in \mathrm{t}_{i}^{*} \mid(\lambda, \alpha)_{i} \in \mathbf{Z}\right.$ for $\left.\forall \alpha \in Q_{i}\right\}$. Thus the corresponding diagram automorphism should be read from the action of $t_{\left(\left(\theta_{i}, \theta_{i}\right) / 2\right) \gamma_{i}}^{(i)}$. Hereafter we shall denote this diagram automorphism simply as $\rho_{\gamma_{i}}^{\mathfrak{h}_{i}}(1 \leqq i \leqq N)$. As for the abelian part, we can implement the action $\sigma_{\gamma_{0}}$ on the infinite sum $\sum L_{\mathrm{ho}}(\hat{\xi})$ by a shift of weight $\widehat{\xi}_{0}$ to $t_{\gamma_{0}}\left(\hat{\xi}_{0}\right)$.

$$
\hat{\xi}_{0} \in Q \frac{\perp}{0}+k_{0} \Lambda 0
$$

Now we can utilize these results in order to investigate the relations among the branching coefficients in (4.1). To do this, we first recall that the isomorphism (4.1) is given by a linear map $\Psi: B_{\hat{\Lambda}, \hat{\xi}}^{(\varepsilon)} \otimes L_{\mathfrak{h}}(\hat{\xi}) \rightarrow \mathscr{F}_{\varepsilon} \otimes L(\hat{\Lambda})$ defined $[\mathrm{TK}]$ by

$$
\Psi: v \otimes J_{a_{1}}\left(-n_{1}\right) \cdots J_{a_{j}}\left(-n_{j}\right)|\widehat{\xi}\rangle \mapsto \widehat{J}_{a_{1}}\left(-n_{1}\right) \cdots \widehat{J}_{a_{j}}\left(-n_{j}\right) v
$$

From now on we fix this isomorphism $\Psi$. Then we can construct the following commuting diagram of isomorphisms; 


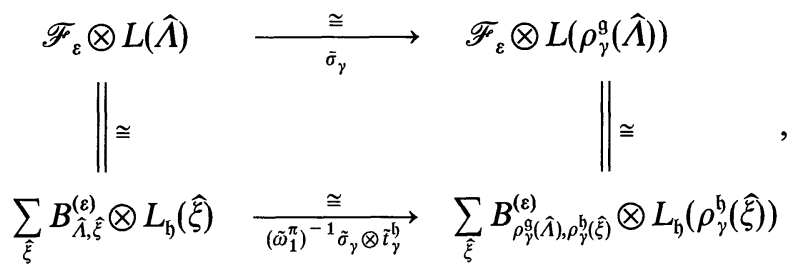

where we defined abbreviations as $\tilde{t}_{\gamma}^{\mathrm{h}} \equiv \tilde{t}_{\gamma_{0}}^{(0)} \otimes \tilde{t}_{\left(\left(\theta_{1}, \theta_{1}\right) / 2\right) \gamma_{1}}^{(1)} \otimes \cdots \otimes \tilde{t}_{\left(\left(\theta_{N}, \theta_{N}\right) / 2\right) \gamma_{N}}^{(N)}$ and $\rho_{\gamma}^{\mathfrak{h}}(\hat{\xi}) \equiv\left(t_{\gamma_{0}}\left(\hat{\xi}_{0}\right), \rho_{\gamma_{1}}^{\mathfrak{h}_{1}}\left(\hat{\xi}_{1}\right), \ldots, \rho_{\gamma_{N}}^{\mathfrak{h}_{N}}\left(\hat{\xi}_{N}\right)\right)$. The element $\tilde{\omega}_{1}^{\pi}$ in the diagram is determined from the element $\tilde{\omega}_{1} \in \tilde{W}_{1}$ which satisfies $t_{\gamma}^{\mathfrak{h}}=\tilde{\omega}_{1} \rho_{\gamma}^{\mathrm{h}}$. The commutativity of the diagram entails the following isomorphism for the branching coefficient modules;

Proposition 4.3. (Field identification relation). For each $\gamma \in Q^{\perp}$, there is an isomorphism of $N=2$ SCA-module;

where $\tilde{t}_{\gamma}^{N=2} \equiv\left(\tilde{\omega}_{1}^{\pi}\right)^{-1} \tilde{\sigma}_{\gamma}$.

$$
\tilde{t}_{\gamma}^{N=2}: B_{\hat{\Lambda}, \hat{\xi}}^{(\varepsilon)} \rightarrow B_{\rho_{\gamma}^{g}(\hat{\Lambda}), \rho_{\gamma}^{\mathrm{b}}(\hat{\xi})}^{(\varepsilon)}
$$

Since we know that $\tilde{\sigma}_{\gamma}$ and $\tilde{\omega}_{1}^{\pi}$ commute with the $N=2 \mathrm{SCA}$, the isomorphism (4.26) tells us the equality among the character formulas for $N=2$ SCA-modules; $\operatorname{ch}\left(B_{\hat{\Lambda}, \hat{\xi}}^{(\varepsilon)}\right)=\operatorname{ch}\left(B_{\rho_{\gamma}^{g}(\hat{\Lambda}), \rho_{\gamma}^{(\xi)}(\hat{\xi})}^{(\varepsilon)}\right)$ This is the reason why we identify the primary fields [BPZ] which represent the $N=2$ SCA-module $B_{\hat{\Lambda}, \hat{\xi}}^{(\varepsilon)}$ and $B_{\rho_{\hat{g}}^{(}(\hat{\Lambda}), \rho_{\hat{\gamma}}^{\mathrm{p}(\hat{\xi})}}^{(\hat{\xi})}$. A rather trivial, but important, field identification follows from the action $\sigma_{\gamma_{0}}$ with $\gamma_{0} \in Q_{0}$. This action entails the following isomorphism;

$$
\mathscr{F}_{\varepsilon} \otimes L(\hat{\Lambda}) \cong \sum_{\hat{\xi} \in \hat{P}(\mathfrak{h})_{+, k} / k_{0} Q_{0}} \sum_{\gamma_{0} \in Q_{0}} \tilde{t}_{\gamma_{0}}^{N=2}\left(B_{\hat{\Lambda}, \hat{\xi}}^{(\varepsilon)} \otimes L_{\mathfrak{h}}\left(t_{\gamma_{0}}(\hat{\xi})\right) .\right.
$$

Note that in this expression we have succeeded in separating the infinite summation in (4.1) into the infinite multiplicities of $N=2 \mathrm{SCA}$-modules.

(4-2) Modular Transformation Properties. Let us give an interpretation for the field identification relation (4.26) from a viewpoint of the modular transformation properties of the characters. Here we define the character for the $N=2 \mathrm{SCA}$ as the branching coefficients which arise when we evaluate the relation (4.1) in terms of the affine characters defined over $\widetilde{P}_{+} \bmod \mathbf{C} \delta[\mathrm{KW}]$ and the product of the theta functions for $\mathscr{F}$ [Fr, Has].

Let us recall $[\mathrm{KP}, \mathrm{KW}]$ that the characters for $\hat{\mathrm{g}}$-modules; $\operatorname{ch}^{\prime}(L(\hat{\Lambda}))\left(\hat{\Lambda} \in P_{+, k}\right)$, transform among themselves under the modular transformations, $S$ and $T$, as

$$
\begin{aligned}
\left.\operatorname{ch}^{\prime}(L(\hat{\Lambda}))\right|_{S} & =\sum_{\hat{\Lambda}^{\prime} \in P+\hat{\Lambda}^{\prime}(c)=k} S_{\hat{\Lambda}, \hat{\Lambda}^{\prime}} \operatorname{ch}^{\prime}\left(L\left(\hat{\Lambda}^{\prime}\right)\right), \\
\left.\operatorname{ch}^{\prime}(L(\hat{\Lambda}))\right|_{T} & =e^{2 \pi i S_{\hat{\Lambda}}} \operatorname{ch}^{\prime}(L(\hat{\Lambda}))
\end{aligned}
$$

where $S_{\hat{\Lambda}} \equiv \frac{(\hat{\Lambda}+\hat{\rho}, \hat{\Lambda}+\hat{\rho})}{2\left(k+g^{*}\right)}-\frac{(\hat{\rho}, \hat{\rho})}{2 g^{*}}$, and the unitary matrix $S_{\hat{\Lambda}, \hat{\Lambda}^{\prime}}\left(=S_{\hat{\Lambda}^{\prime}, \hat{\Lambda}}\right)$ is given by

$$
S_{\hat{\Lambda} \hat{\Lambda}^{\prime}}=i^{|\Delta+|}|P / M|^{-1 / 2}\left(k+g^{*}\right)^{-1 / 2} \sum_{\omega \in W} \operatorname{det} \omega e^{-\left(2 \pi i / k+g^{*}\right)\left(\hat{\Lambda}+\hat{\rho}, \omega\left(\hat{\Lambda}^{\prime}+\hat{\rho}\right)\right)}
$$

To apply this property to $\hat{\mathfrak{h}}$-modules, we must deal with the infinite multiplicities associated with the abelian part $\hat{\mathfrak{h}}_{0}[\mathrm{TK}]$. Before going into this subject, let us 
first discuss about the fermionic part. As for the character of the $\mathscr{F}_{\varepsilon}$, it is known that there are four possible types; $\operatorname{ch}\left(\mathscr{F}_{\varepsilon}^{ \pm}\right)$, where the minus sign means the insertion of the operator $(-1)^{J_{f}(0)}$ into the trace for the characters while the plus sign does not. These four possibilities are connected to the characters of the level 1 representations of $\hat{s o}(2 m)\left(m \equiv \operatorname{dim} m_{+}\right)$as $\operatorname{ch}\left(\mathscr{F} \frac{ \pm}{\mathrm{NS}}\right)=\operatorname{ch}^{\prime}\left(L\left(\hat{\Lambda}_{0}\right)\right) \pm \operatorname{ch}^{\prime}\left(L\left(\hat{\Lambda}_{1}\right)\right)$, $\operatorname{ch}\left(\mathscr{F}_{\mathrm{R}}^{ \pm}\right)=\operatorname{ch}^{\prime}\left(L\left(\hat{\Lambda}_{m}\right)\right) \pm \operatorname{ch}^{\prime}\left(L\left(\hat{\Lambda}_{m-1}\right)\right)$. Thus we see that $\operatorname{ch}\left(\mathscr{F}_{\varepsilon}^{ \pm}\right)$close among themselves under the modular transformation, which we denote as $f_{\left(\varepsilon^{\prime}, b\right)}^{(\varepsilon, a)}$ for the $S$-transformation and as $t_{f}^{(\varepsilon, a)}$ for the $T$-transformation with $\varepsilon, \varepsilon^{\prime}=0$ or $1 / 2$ and $a, b= \pm$.

We can now express the decomposition (4.1) in terms of the characters as

$$
\operatorname{ch}\left(\mathscr{F}_{\varepsilon}^{ \pm}\right) \cdot \operatorname{ch}^{\prime}(L(\hat{\Lambda}))=\sum_{\hat{\xi} \in \hat{P}(\hat{h})+, k} \operatorname{ch}\left(B_{\hat{\Lambda}, \hat{\xi}}^{(\varepsilon, \pm)}\right) \cdot \operatorname{ch}^{\prime}\left(\hat{L}\left(\hat{\xi}_{0}\right)\right) \cdot \operatorname{ch}^{\prime}\left(\hat{L}\left(\hat{\xi}_{1}\right)\right) \cdots \operatorname{ch}^{\prime}\left(\hat{L}\left(\hat{\xi}_{N}\right)\right)
$$

with

$$
\operatorname{ch}^{\prime}\left(\hat{L}\left(\hat{\xi}_{0}\right)\right) \equiv e\left(-\frac{\left(\xi_{0}, \xi_{0}\right)}{2 k_{0}} \delta+\hat{\xi}_{0}\right) / \eta^{\mathrm{dim} \xi_{0}},
$$

where $\eta$ is the Dedekind $\eta$-function and $e(\lambda)(h) \equiv e^{\langle\lambda, h\rangle}\left(\lambda \in \mathrm{t}^{*}, h \in \mathrm{t}\right)$. When we recall the relation (4.27) and note that $\tilde{\sigma}_{\gamma_{0}} J_{f}(0)\left(\tilde{\sigma}_{\gamma_{0}}\right)^{-1}=J_{f}(0)-2\left(\rho_{2}, \gamma_{0}\right)$ and $2\left(\rho_{2}, \gamma_{0}\right) \in 2 \mathbf{Z}$, we deduce that the infinite multiplicities of $N=2$ SCA-module are summarized into the theta function;

$$
\begin{aligned}
\operatorname{ch}\left(\mathscr{F}_{\varepsilon}^{ \pm}\right) \cdot \operatorname{ch}^{\prime}(L(\hat{\Lambda})) & =\sum_{\hat{\xi} \in \hat{P}(\hat{h})+, \mathbf{k} / k_{0} Q_{0}} \operatorname{ch}\left(B_{\hat{\Lambda}, \hat{\xi}}^{(\varepsilon, \pm)}\right) \cdot \Theta_{\hat{\xi}_{0}}^{Q_{0}} \cdot \operatorname{ch}^{\prime}\left(\hat{L}\left(\hat{\xi}_{1}\right)\right) \cdots \operatorname{ch}^{\prime}\left(\hat{L}\left(\hat{\xi}_{N}\right)\right), \\
& \equiv \sum_{\hat{\xi} \in \hat{P}(\hat{h})_{+, \mathbf{k}} / k_{0} Q_{0}} b_{\hat{\Lambda}, \hat{\xi}}^{(\varepsilon, a)} \chi(\hat{L}(\hat{\xi})),
\end{aligned}
$$

where

$$
\Theta_{\hat{\xi}_{0}}^{Q_{0}} \equiv e\left(-\frac{\left(\xi_{0}, \xi_{0}\right)}{2 k_{0}} \delta\right) \sum_{\gamma_{0} \in Q_{0}} e\left(t_{\gamma_{0}}\left(\widehat{\xi}_{0}\right)\right) / \eta^{\mathrm{dim} \mathfrak{H}_{0}} .
$$

In this form we can apply the results (4.28-30).

Due to the considerations above, we can derive the modular transformation properties of $b_{\hat{\Lambda}, \hat{\xi}}^{(\varepsilon, a)}$ as;

$$
\begin{aligned}
&\left.b_{\hat{\Lambda}, \xi}^{(\varepsilon, a)}\right|_{S}=\sum_{\substack{\hat{\Lambda}^{\prime}, \xi^{\prime} \\
\left(\hat{\xi}^{\prime}, b\right)}} f_{\left(\varepsilon^{\prime}, b\right)}^{(\varepsilon, a)} S_{\hat{\Lambda}, \hat{\Lambda}^{\prime}} S_{\hat{\xi}, \xi^{\prime}}^{*} b_{\hat{\Lambda}^{\prime}, \xi^{\prime}}^{\left(\varepsilon^{\prime}, b\right)}, \\
&\left.b_{\hat{\Lambda}, \hat{\xi}}^{(\varepsilon, a)}\right|_{T}=\exp \left\{2 \pi i\left(t_{f}^{(\varepsilon, a)}+S(\mathfrak{g})_{\hat{\Lambda}}-S(\mathfrak{h})_{\hat{\xi}}\right)\right\} b_{\hat{\Lambda}, \hat{\xi}}^{(\varepsilon, a)},
\end{aligned}
$$

where $\hat{\xi}, \hat{\xi}^{\prime} \in P(\mathfrak{h})_{+, \mathbf{k}} / k_{0} Q_{0}$.

Now we can verify;

Proposition 4.4. The transformation matrices satisfy the following relations;

$$
\begin{aligned}
& f_{\left(\varepsilon^{\prime}, b\right)}^{(\varepsilon, a)} S_{\rho_{\hat{\gamma}}^{\mathrm{g}}(\hat{\Lambda}), \hat{\Lambda}^{\prime}} S_{\rho_{\gamma}^{\mathrm{b}}(\hat{\xi}), \hat{\xi}^{\prime}}^{*}=f_{\left(\varepsilon^{\prime}, b\right)}^{(\varepsilon, a)} S_{\hat{\Lambda}, \hat{\Lambda}^{\prime}} S_{\xi \xi^{\prime}}^{*} \quad \text { for } S \text { transf., } \\
& \exp \left\{2 \pi i\left(t_{f}^{(\varepsilon, a)}+S(\mathfrak{g})_{\rho_{\gamma}^{g}(\hat{\Lambda})}-S(\mathfrak{h})_{\rho_{\rho}^{g}(\hat{\xi})}\right)\right\} \\
& =\exp \left\{2 \pi i\left(t_{f}^{(\varepsilon, a)}+S(\mathfrak{g})_{\hat{\Lambda}}-S(\mathfrak{h})_{\hat{\xi}}\right)\right\} \quad \text { for T transf. }
\end{aligned}
$$

To prove this statement, we note that the $S$-matrix has a property (Eq. (2.2.13) 
in ref. [KW] also see ref. [Gep]);

$$
S_{\sigma(\hat{\Lambda}), \hat{\Lambda}^{\prime}}=e^{2 \pi i\left(\Lambda^{\prime}, \varepsilon\right)} S_{\hat{\Lambda}, \hat{\Lambda}^{\prime}}
$$

for a diagram automorphism $\sigma=t_{\varepsilon} \sigma_{W} \in \tilde{W}_{0}^{+}, \sigma_{W} \in W$. Then the equalities immediately follow from the following relations;

$$
\begin{aligned}
& S_{\rho_{\gamma}^{g}(\hat{\Lambda}), \hat{\Lambda}^{\prime}} S_{\rho_{\gamma}^{\mathrm{y}}(\hat{\xi}), \hat{\xi}^{\prime}}^{*}=e^{2 \pi i\left(\hat{\Lambda}^{\prime}, \gamma\right)-2 \pi i\left(\hat{\xi}^{\prime}, \gamma\right)} S_{\hat{\Lambda}, \hat{\Lambda}^{\prime}} S_{\hat{\xi} \hat{\xi}^{\prime}}^{*}, \\
& \Lambda^{\prime}-\xi^{\prime} \in Q, \quad \rho_{\gamma}^{\mathrm{g}}(\hat{\rho})=\hat{\rho}, \quad \rho_{\gamma}^{\mathrm{b}}\left(\hat{\rho}_{1}\right)=\hat{\rho}_{1},
\end{aligned}
$$

where

$$
\hat{\rho}_{1} \equiv \frac{1}{2} \sum_{\alpha \in \Delta(\mathfrak{h})_{+}} \alpha+\sum_{i} h_{i}^{*} \Lambda_{(0) i} .
$$

Proposition 4.4 ensures the compatibility of the field identification relation (4.26) with the modular transformation properties (4.33).

(4-3) R↔NS Spectral Flow. Here we will formulate an isomorphism among the branching coefficient modules with different fermionic boundary conditions. This isomorphism is the origin of the space-time supersymmetry in the compactified string theory and plays an important role in physics [BDFM].

Now let us define an automorphism of the Clifford algebra $\sigma_{s}^{f}(s \in \mathbf{Z} / 2)$ by

$$
\sigma_{s}^{f}\left(\psi^{\alpha}(r)\right)=\psi^{\alpha}(r+s), \quad \sigma_{s}^{f}\left(\psi^{\bar{\alpha}}(r)\right)=\psi^{\bar{\alpha}}(r-s)
$$

for all $\alpha \in \Delta\left(\mathrm{m}_{+}\right), \bar{\alpha} \in \Delta\left(\mathrm{m}_{-}\right) . \sigma_{s}^{f}$ act trivially on the $\hat{\mathrm{g}}$-currents. Then the following action of $\sigma_{s}^{f}$ on the $N=2$ SCA and $\hat{\mathfrak{h}}$-currents follows from simple calculations;

Proposition 4.5. (R↔NS spectral flow). The actions of $\sigma_{s}^{f}(s \in \mathbf{Z} / 2)$ defines the following automorphisms;

i) $N=2 \mathrm{SCA}$;

$$
\begin{gathered}
\sigma_{s}^{f}\left(T(n)_{\mathrm{R}}\right)=T(n)_{s}+\{s\} J(n)_{s}+\frac{c_{v}}{6}\{s\}^{2} \delta_{n, 0}, \\
\sigma_{s}^{f}\left(G^{ \pm}(r)_{\mathrm{R}}\right)=G^{ \pm}(r \pm s)_{s}, \quad \sigma_{s}^{f}\left(J(n)_{R}\right)=J(n)_{s}+\frac{c_{v}}{3}\{s\} \delta_{n, 0} \\
\sigma_{s}^{f}\left(T(n)_{\mathrm{NS}}\right)=T(n)_{s+1 / 2}+[s] J(n)_{s+1 / 2}+\frac{c_{v}}{6}[s]^{2} \delta_{n, 0}, \\
\sigma_{s}^{f}\left(G^{ \pm}(r)_{\mathrm{NS}}\right)=G^{ \pm}(r \pm s)_{s+1 / 2}, \quad \sigma_{s}^{f}\left(J(n)_{\mathrm{NS}}\right)=J(n)_{s+1 / 2}+\frac{c_{v}}{3}[s] \delta_{n, 0},
\end{gathered}
$$

ii) $\hat{\mathfrak{h}}$-currents;

$$
\begin{aligned}
\sigma_{s}^{f}\left(\hat{J}_{\alpha}(n)_{\mathrm{R}}\right) & =\hat{J}_{\alpha}(n)_{s}, \quad \sigma_{s}^{f}\left(\hat{J}_{\alpha}(n)_{\mathrm{NS}}\right)=\hat{J}_{\alpha}(n)_{s+1 / 2}, \\
\sigma_{s}^{f}\left(\hat{H}_{\gamma}(n)_{\mathrm{R}}\right) & =\hat{H}_{\gamma}(n)_{s}-\left(2 \rho_{2}, \gamma\right)\{s\} \delta_{n, 0}, \\
\sigma_{s}^{f}\left(\hat{H}_{\gamma}(n)_{\mathrm{NS}}\right) & =\hat{H}_{\gamma}(n)_{s+1 / 2}-\left(2 \rho_{2}, \gamma\right)[s] \delta_{n, 0},
\end{aligned}
$$

where the subscripts $s$ and $s+1 / 2$ indicates the types of the operators, $\mathrm{R}$ or $\mathrm{NS}$, 
according to their value $\mathbf{Z}$ or $\mathbf{Z}+\frac{1}{2}$, respectively, and $[s]$ and $\{s\}$ are integers determined from the relation $s-1<[s] \leqq s \leqq\{s\}<s+1$.

Here we note that the actions i) are different from the conventional one (e.g. (1.16) in ref. [LVW]) in that the parameter of the flow appears as an integer in the right-hand side of $i)$. This is, again, the reflection of the different choice of the normal ordering prescription as noted in the final remark in Sect. 2.

We can easily implement these automorphisms on $\mathscr{F}_{0} \oplus \mathscr{F}_{1 / 2}$ as follows;

Proposition 4.6. There exist isomorphisms $\tilde{\sigma}_{s}^{f}(s \in \mathbf{Z} / 2)$ on $\mathscr{F}_{0} \oplus \mathscr{F}_{1 / 2}$ which implement the action $\sigma_{s}^{f}$. They are defined by

$$
\tilde{\sigma}_{s}^{f}|0\rangle_{\mathbf{R}}= \begin{cases}\prod_{\alpha \in \Delta\left(\mathrm{m}_{+}\right)} \psi^{\alpha}(s+1) \cdots \psi^{\alpha}(s-\{s\}) \tilde{\sigma}_{s-\{s\}}^{f}|0\rangle_{\mathbf{R}} & \text { for } s \leqq-1 \\ \prod_{\bar{\alpha} \in \Delta\left(\mathrm{m}_{-}\right)} \psi^{\bar{\alpha}}(-s) \cdots \psi^{\bar{\alpha}}(-s+\{s\}-1) \tilde{\sigma}_{s-\{s\}}^{f}|0\rangle_{\mathbf{R}} & \text { for } s>0,\end{cases}
$$

and

$$
\tilde{\sigma}_{s}^{f}|0\rangle_{\mathrm{NS}}= \begin{cases}\prod_{\alpha \in \Delta\left(\mathfrak{m}_{+}\right)} \psi^{\alpha}\left(s+\frac{1}{2}\right) \cdots \psi^{\alpha}\left(s-[s]-\frac{1}{2}\right) \tilde{\sigma}_{s-[s]}^{f}|0\rangle_{\mathrm{NS}} & \text { for } \quad s \leqq-1 \\ \prod_{\bar{\alpha} \in \Delta\left(\mathrm{m}_{-}\right)} \psi^{\bar{\alpha}}\left(-s+\frac{1}{2}\right) \cdots \psi^{\bar{\alpha}}\left(-s+[s]-\frac{1}{2}\right) \tilde{\sigma}_{s-[s]}^{f}|0\rangle_{\mathrm{NS}} & \text { for } \quad s>0,\end{cases}
$$

where $\tilde{\sigma}_{-1 / 2}^{f}|0\rangle_{\mathrm{R}} \equiv|0\rangle_{\mathrm{NS}}$ and $\tilde{\sigma}_{1 / 2}^{f}|0\rangle_{\mathrm{NS}} \equiv|0\rangle_{\mathrm{R}}$.

Since the action on $\hat{\mathfrak{h}}$-currents coincides with that of the translation, which acts only on the abelian part $\mathfrak{h}_{0}$, we deduce the isomorphism;

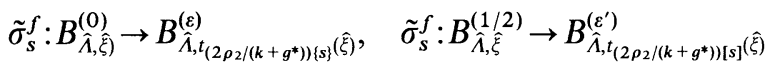

with $\varepsilon=0$ or $1 / 2\left(\varepsilon^{\prime}=1 / 2\right.$ or 0$)$ according to $s \in \mathbf{Z}$ or $\mathbf{Z}+\frac{1}{2}$, respectively. In particular, if we substitute $s=-1 / 2$, then we obtain

$$
\tilde{\sigma}_{-1 / 2}^{f}: B_{\hat{\Lambda}, \hat{\xi}}^{(\mathrm{R})} \rightarrow B_{\hat{\Lambda}, \hat{\xi}}^{(\mathrm{NS})}
$$

Since $\tilde{\sigma}_{-1 / 2}^{f}$ defines the co-chain map; $\tilde{\sigma}_{-1 / 2}^{f} G^{ \pm}(0)_{\mathrm{R}}-G^{ \pm}\left(\mp \frac{1}{2}\right)_{\mathrm{NS}} \tilde{\sigma}_{-1 / 2}^{f}=0$, between $C^{*}\left(\mathrm{~m}_{+} \otimes \mathbf{C}\left[t, t^{-1}\right], L(\hat{\Lambda})\right)_{0}$ and $C^{*}\left(\mathrm{~m}_{+} \otimes \mathbf{C}\left[t, t^{-1}\right], L(\hat{\Lambda})\right)_{1 / 2}$, we obtain the following isomorphism of the harmonic forms:

$$
\tilde{\sigma}_{-1 / 2}^{f}: \mathbf{H}^{*}\left(\mathrm{~m}_{+} \otimes \mathbf{C}\left[t, t^{-1}\right], L(\hat{\Lambda})\right)_{\mathbf{R}} \rightarrow \mathbf{H}^{*}\left(\mathrm{~m}_{+} \otimes \mathbf{C}\left[t, t^{-1}\right], L(\hat{\Lambda})\right)_{\mathrm{NS}} .
$$

This isomorphism is the one which we have promised to show in previous section. (4-4) Charge Conjugation. In this subsection, we formulate, for later use, a somewhat different symmetry: the charge conjugation automorphism.

Definition 4.7. (Charge conjugation). We define the charge conjugation automorphisms, $\mathscr{C}$, for the Clifford algebra and the affine Lie algebra $\hat{\mathfrak{g}}$ by

$$
\mathscr{C}\left(\psi^{\alpha}(r)\right)=-\psi^{-\alpha}(r) \quad\left(\alpha \in \Delta\left(\mathrm{m}_{+}\right) \sqcup \Delta\left(\mathrm{m}_{-}\right)\right)
$$

and

$$
\mathscr{C}\left(J^{\alpha}(n)\right)=-J^{-\alpha}(n), \quad \mathscr{C}\left(H_{\alpha}(n)\right)=H_{-\alpha}(n) \quad(\alpha \in \Delta),
$$

respectively. 
Then the following results follow from simple calculations;

Proposition 4.8. The charge conjugation automorphisms (4.49) and (4.50) are implementable on the Fock space $\mathscr{F}_{\varepsilon}$ and $\hat{\mathrm{g}}$-modules $L_{k}$ by

and

$$
\mathscr{C}^{\pi}(|0\rangle)= \begin{cases}\prod_{\alpha \in \Delta\left(\mathfrak{m}_{+}\right)} \psi^{\alpha}(0)|0\rangle & \text { for } \\ |0\rangle & \text { for } \varepsilon=0\end{cases}
$$

$$
\mathscr{C}^{\pi}(|\hat{\Lambda}\rangle)=\omega_{\text {long }}^{\pi}\left|\hat{\Lambda}^{c}\right\rangle, \quad \hat{\Lambda} \in \hat{P}_{+, k},
$$

with $\hat{\Lambda}^{c} \equiv-\omega_{\text {long }}(\hat{\Lambda})$, respectively. Where $\omega_{\text {long }}$ in (4.52) is the longest element of the classical Weyl group of $\mathfrak{g}$.

The following results are also consequences of the straightforward calculations using the formula (2.39);

Proposition 4.7. The charge conjugation operations (4.49), (4.50) act on the $\hat{\mathfrak{h}}$-current algebra and the $N=2$ SCA as an algebra automorphism in following way:

i) for $\hat{\mathfrak{h}}$-currents

$$
\mathscr{C}\left(\hat{J}_{\beta}(n)\right)=-\hat{J}_{-\beta}(n), \quad \mathscr{C}\left(\hat{H}_{\beta}(n)\right)=\hat{H}_{-\beta}(n) \quad(\beta \in \Delta(\mathfrak{h})),
$$

ii) for $N=2 \operatorname{SCA}(\varepsilon=0)$

$$
\begin{aligned}
& \mathscr{C}\left(G^{ \pm}(r)\right)=G^{\mp}(r), \\
& \mathscr{C}(T(n))=T(n)-J(n)+\frac{c_{v}}{6} \delta_{n, 0}, \\
& \mathscr{C}(J(n))=-J(n)+\frac{c_{v}}{3} \delta_{n, 0},
\end{aligned}
$$

iii) for $N=2 \operatorname{SCA}(\varepsilon=1 / 2)$

$$
\begin{aligned}
\mathscr{C}\left(G^{ \pm}(r)\right) & =G^{ \pm}(r), \quad \mathscr{C}(T(n))=T(n), \\
\mathscr{C}(J(n)) & =-J(n) .
\end{aligned}
$$

We easily see that the algebra automorphism (4.53) is implemented as (4.52) on the extended $\hat{\mathfrak{h}}$-module $L_{\mathfrak{h}} \equiv \sum_{i} L_{k_{i}}$. In this case we must use the longest element $\omega_{\text {long }}^{\mathfrak{h}_{i}}$ of each $W\left(\mathfrak{h}_{i}\right)(1 \leqq i \leqq N)$. This fact implies that there exists an isomorphism

$$
\tilde{\mathscr{C}}^{\pi} \equiv \prod_{i=1}^{N}\left(\omega_{\text {long }}^{h_{i}}\right)^{-1} \mathscr{C}^{\pi}: B_{\hat{\Lambda}, \hat{\xi}}^{(\varepsilon)} \rightarrow B_{\hat{\Lambda}^{c}, \hat{\xi}^{c}}^{(\varepsilon)}, \quad \hat{\xi}^{c} \equiv\left(-\widehat{\xi}_{0}, \widehat{\xi}_{1}^{c}, \ldots, \hat{\xi}_{N}^{c}\right),
$$

which implements the automorphisms (4.54)-(4.56), (4.57).

\section{Poincaré Polynomial for $N=2$ SCFT}

In previous sections, we have studied in detail the $N=2$ super-GKO construction and its cohomological interpretations. In this section the results obtained there will be unified from a viewpoint of the $N=2$ SCFTs and the Poincare polynomials 
associated with them. The meaning of the Poincare polynomial, especially its geometrical meaning, still remains to be clarified in future investigations.

(5-1) Poincaré Polynomial and Its Physical Background. The $N=2$ SCFT may be stated, as one definition, as the modular invariant partition functions constructed from the characters $b_{\hat{\Lambda}, \tilde{\xi}}^{(\varepsilon, a)}(\tau)$ for the branching coefficient modules $B_{\hat{\Lambda}, \hat{\xi}}^{\varepsilon}$. It is known that there are many types of the modular invariants [CIZ, Kat]. Though such a variety may always exist for the $N=2 \mathrm{SCFT}$, we restrict our arguments, for simplicity, to the diagonal modular invariant only;

$$
Z(\tau)=\frac{1}{\mathscr{N}} \sum_{\substack{(\varepsilon, a) ; \hat{\Lambda} \in \hat{P}_{+, k} \\ \xi \in \hat{P}(b)+, \mathbf{k}^{\prime / k} Q_{0}}}\left|b_{\hat{\Lambda}, \xi}^{(\varepsilon, a)}(\tau)\right|^{2},
$$

with $\mathscr{N}$ representing a constant which we read from the multiplicity of the vacuum (the module with both the conformal dimension and the charge being equal to zero) among the branching coefficient modules. As formulated in Proposition 4.3 in previous section, the multiplicity of the branching coefficients as $N=2$ SCAmodule follows from the action of $\gamma \in Q^{\perp}$ on $L_{k}$. When the action $\gamma \in Q^{\perp}$ has a fixed point, the definition of the $N=2$ SCFT by (5.1) becomes troublesome because the fractional coefficient appears in the partition function. For the time being, we restrict ourselves to the case that there does not appear such fixed points. We will return to this problem later in this subsection.

The characteristic feature of the $N=2$ SCFT is that some class of the theory defined by (5.1) admits a description by Landau-Ginzburg (L-G) effective Lagrangian [VW] [Mar]. This observation is based on the fact that the set of the relevant operators contained in the $\mathrm{L}-\mathrm{G}$ theory and the primary fields that are labeled by the weights representing the cohomology elements in (5.1) coincides with each other in their charges and conformal dimensions (in fact the charge is two times the conformal dimension for the cohomology elements). The primary fields in this correspondence are named as the chiral primary fields in ref. [LVW]. One of our interests here is to investigate, from the physical point of view, how large classes of the $N=2 \mathrm{SCFT}$ admit such a L-G description [LVW].

To this aim we define a certain Poincaré polynomial associated with each $N=2$ SCFT. This Poincaré polynomial might have also mathematical importance since it will turn out that it is defined in deep relation with the Poincaré polynomial of the semi-infinite flag manifolds.

Let us recall that the $\mathrm{L}-\mathrm{G}$ theory with $N=2$ supersymmetry has the following features which follows from the non-renormalization theorem: i) The theory at critical point is described essentially by the super-potential $W\left(\Phi_{1}, \ldots, \Phi_{n}\right)$, where $\Phi_{i}(1 \leqq i \leqq n)$ represents the chiral superfield. ii) The super-potential must have the quasihomogeneous property such that $W\left(\lambda^{\omega_{1}} \Phi_{1}, \ldots, \lambda^{\omega_{n}} \Phi_{n}\right)=\lambda^{d} W\left(\Phi_{1}, \ldots, \Phi_{n}\right)$ in order to realize the theory at critical point. iii) The set of the relevant operators in the $\mathrm{L}-\mathrm{G}$ theory is expected as the ring $J$,

$$
J=\frac{\mathbf{C}\left[\Phi_{1}, \ldots, \Phi_{n}\right]}{\left[\partial W / \partial \Phi_{1}, \ldots, \partial W / \partial \Phi_{n}\right]} .
$$

If the order of the ring $J$ is finite dimensional, we can define the Poincare polynomial for a given $\mathrm{L}-\mathrm{G}$ theory by

$$
P_{J}(t)=\operatorname{Tr}_{J} t^{Q}
$$


with the charge operator $Q: J \rightarrow J$ defined by $Q\left(\Phi_{i}\right)=\omega_{i} \Phi_{i}(1 \leqq i \leqq n)$.

Generally, a formal power series $f \in \mathfrak{M} \subset \mathbf{C}\left[x_{1}, \ldots, x_{n}\right]$ is called as non-degenerate near 0 if there exists a natural number $m$ such that $\mathfrak{M} \supset \mathfrak{U} \supset \mathfrak{M}^{m}$, where $\mathfrak{M}$ represents the maximal ideal of a local ring $\mathbf{C}\left[x_{1}, \ldots, x_{n}\right]$ and $\mathfrak{U} \equiv\left(\partial f / \partial x_{1}, \ldots, \partial f / \partial x_{n}\right)$. Just the same way as $J$ in (5.2), the local algebra of $f$ is defined by $R_{f} \equiv \mathbf{C}\left[x_{1}, \ldots, x_{n}\right] /$ $\left(\partial f / \partial x_{1}, \ldots, \partial f / \partial x_{n}\right)$. Then the following property is known:

Theorem 5.1. (V. I. Arnold [AGV], Theorem on p. 199, I). Let $f$ be a nondegenerate quasihomogeneous polynomial such that $f\left(\lambda^{\omega_{1}} x_{1}, \ldots, \lambda^{\omega_{n}} x_{n}\right)=\lambda^{d} f\left(x_{1}, \ldots, x_{n}\right)$ $\left(\lambda \in \mathbf{C}^{*} ; \omega_{i}, d \in \mathbf{Z}_{>0}\right)$. Then we have

$$
\chi_{f}(t)=\sum \mu_{i} t^{i}=\prod_{i=1}^{n} \frac{\left(1-t^{d-\omega_{i}}\right)}{\left(1-t^{\omega_{i}}\right)},
$$

where $\mu_{i}$ is the number of basis monomials in $R_{f}$ with quasidegree $i / d$.

In this theorem, the non-degenerate property of $f$ ensures that the number of the basis monomials in $R_{f}$ in finite.

From the above theorem, we see that every Poincaré polynomial $P_{J}(t)$ can be cast into the product form as (5.4) for the $N=2 \mathrm{~L}-\mathrm{G}$ theory with non-degenerate quasihomogeneous superpotential $W\left(\Phi_{1}, \ldots, \Phi_{n}\right)$.

Keeping this background for the L-G theory in mind, we proceed to

Definition 5.2. (Poincaré polynomial). We define for each $N=2$ SCFT a Poincaré polynomial by

$$
P(t)=\sum_{\chi \in \mathscr{I}} t^{Q(\chi)}
$$

where $\mathscr{I}$ is a quotient

$$
\mathscr{I} \equiv P_{+, k} \times \tilde{W}^{1} / \sim,
$$

with the equivalence relation, $\sim$, defined by the field identification (4.26), that is, $(\hat{\Lambda}, \tilde{\omega}) \sim\left(\hat{\Lambda}^{\prime}, \tilde{\omega}^{\prime}\right)$ if there exist $\gamma \in Q^{\perp}$ such that $t_{\gamma}^{N=2}: B_{\hat{\Lambda}, \hat{\xi}_{\tilde{\omega}}} \cong B_{\hat{\Lambda}^{\prime}, \hat{\xi}_{\tilde{\omega}^{\prime}}}$. The charge $Q(\chi)$ is determined as an eigenvalue of the charge operator $J(0)$ for $\left|\hat{\Lambda}, \hat{\xi}_{\tilde{\sigma}}\right\rangle \in$ $H_{\varepsilon}^{*}\left(\mathrm{~m}_{+} \otimes \mathbf{C}\left[t, t^{-}\right], L(\widehat{\Lambda})\right)$

$$
Q(\hat{\Lambda}, \tilde{\sigma}) \equiv \frac{2\left(\rho_{2}, \xi_{\tilde{\sigma}}\right)}{k+g^{*}}+\tilde{l}(\tilde{\sigma})
$$

and does not depend on the $\varepsilon$.

Owing to the correspondence between the chiral primary fields and the cohomology elements, the Poincaré polynomial defined above should be identified with (5.3) in the $\mathrm{L}-\mathrm{G}$ theory. Then from Theorem 5.1, the Poincaré polynomial (5.5) must be written in the product form as (5.4). This is a very non-trivial property and should be tested for each choice of $(\mathfrak{g}, \mathfrak{h})$. This will be done for specific models in the latter half of this section. In the following, we study the general structure of the Poincaré polynomials utilizing the results obtained in the previous sections.

Before closing this subsection, we must mention about the invalidity of the definition (5.1) when the action $\gamma \in Q^{\perp}$ on $L_{k}$ has fixed points as remarked at the beginning. There are some conjectures that in this case the $N=2 \mathrm{SCA}$ extends to some larger algebra with higher symmetry [MS2, SY]. However, how we should 
understand this phenomenon is still unsolved and is an important problem to reveal the coset conformal field theories. So we define the Poincare polynomial (5.5) also for the case with fixed points. Though physical meanings of our definition (5.5) might depend on the resolution of the fixed point problem, it would still be meaningful to reveal the general structures of the Poincare polynomial (5.5).

(5-2) General Structure of the Poincaré Polynomial. According to the results (Theorem 3.8) on $H^{*}\left(\mathrm{~m}_{+} \otimes \mathbf{C}\left[t, t^{-1}\right], L(\hat{\Lambda})\right)$, we can write the cohomology elements bijectively in terms of the subset $\widetilde{W}^{1}$ of the affine Weyl group for $\tilde{\mathfrak{g}}$. This subset $\tilde{W}^{1}$ is revealed, in previous section, to have the structure (3.36),

$$
\tilde{W}^{1}=\tilde{\mathfrak{D}} \cdot W^{1} .
$$

Now we show that

Proposition 5.3. For each $\tilde{\rho}\left(=\omega^{-1} t_{\gamma}\right) \in \tilde{\mathfrak{D}}$, there exists an isomorphism

$$
\tilde{t}_{\gamma}^{N=2}: B_{\hat{\Lambda}, \hat{\xi}_{\tilde{\omega}}}^{(\varepsilon)} \rightarrow B_{\hat{\Lambda}, \hat{\xi}_{\tilde{\rho}(\hat{)})}}^{(\varepsilon)}
$$

That is, we have $(\hat{\Lambda}, \tilde{\omega}) \sim(\hat{\Lambda}, \tilde{\rho} \tilde{\omega})$ for $\forall \tilde{\omega} \in \tilde{W}^{1}$.

To see this property, compare the definition of $\tilde{\mathfrak{D}}$ in (3.34) with the definition $W_{0}^{+}$in (4.10). Then we easily deduce that the group $\tilde{\mathfrak{D}}$ is the Dynkin diagram automorphisms for $\widetilde{\mathfrak{h}}_{i}(1 \leqq i \leqq N)$ which are contained in the affine Weyl group $\tilde{W}$. Then we can verify that the following relations, $\bmod \mathbf{C} \delta$, hold;

$$
\widehat{\xi}_{\tilde{\rho} \tilde{\omega}, i}= \begin{cases}t_{\gamma_{0}}\left(\hat{\xi}_{\tilde{\omega}, 0}\right) & (i=0) \\ \rho_{\gamma_{i}}^{h_{i}}\left(\hat{\xi}_{\tilde{\omega}, i}\right) & (1 \leqq i \leqq N),\end{cases}
$$

for each element $\tilde{\rho}=\omega^{-1} t_{\gamma} \in \tilde{\mathfrak{D}}$. On the other hand we know that $\tilde{\rho} \in \tilde{\mathfrak{D}} \leqq \tilde{W}$ acts on the $\mathfrak{g}$-module $L(\hat{\Lambda})$. Thus we see that there is the field identification isomorphism $\tilde{t}_{\gamma}^{N=2}: B_{\hat{\Lambda}, \hat{\xi}_{\tilde{\omega}}}^{(\varepsilon)} \rightarrow B_{\hat{\Lambda}, \hat{\xi}_{\tilde{\rho} \tilde{\omega}}}^{(\varepsilon)}$

Combining the above result to the structure of the subset $\tilde{W}^{1}$ in (5.8), we obtain the following equivalence relation for $\sigma \in W^{1}$.

$$
\left(\hat{\Lambda}, \tilde{\sigma}^{\prime}\right) \sim(\tilde{\Lambda}, \sigma), \quad \tilde{\sigma}^{\prime} \in \tilde{\mathfrak{D}} \sigma .
$$

This property tells us that the Poincaré polynomial (5.5) is determined, in fact, from the finite set $P_{+, k} \times W^{1}$ rather than the infinite set $P_{+, k} \times \tilde{W}^{1}=$ $P_{+, k} \times T_{0} \cdot \mathfrak{D} \cdot W^{1}$ (see (3.39)). We shall see later, using some examples, that a further reduction of the set $P_{+, k} \times W^{1}$ follows from the isomorphisms $\tilde{t}_{\gamma}^{N=2}\left(\gamma \in Q^{\perp}, \gamma \notin M\right)$. These isomorphisms are related to the diagram automorphisms of $\mathfrak{g}$ as we have shown below (4.9) that $Q^{\perp} / M \cong \widetilde{W}_{0}^{+}$.

Next we show

Proposition 5.4. The Poincaré polynomial (5.5) has a certain duality property;

$$
P(t)=t^{\left(c_{v} / 3\right)} P\left(\frac{1}{t}\right) .
$$

To formulate this property, we first note that the Poincare polynomial can be written as

$$
P(t)=\operatorname{Tr}_{3 R} t^{J(0)}
$$


where $\mathscr{R} \equiv \sum_{\hat{A} \hat{\alpha} \in \mathscr{A}} \mathbf{C}\left|\hat{\Lambda}, \xi_{\tilde{\sigma}}\right\rangle \subset \mathscr{F} \otimes L_{k}$. Then we can verify that the charge conjugation operator $\widetilde{\mathscr{C}}^{(\hat{\lambda}, \tilde{\sigma}) \in \mathscr{F}}$ acts on the space of the harmonic cocycles and that the operators $\tilde{\mathscr{C}}^{\pi}$ and $\tilde{t}_{\gamma}^{N=2}$ commute with each other on the space of the harmonic cocycles. From these facts, we realize that $\tilde{\mathscr{C}}^{\pi}$ acts on the vector space $\mathscr{R}$. Utilizing this property we have

$$
P(t)=\operatorname{Tr}_{\mathscr{R}} \tilde{\mathscr{C}}^{\pi} t^{J(0)}\left(\tilde{\mathscr{C}}^{\pi}\right)^{-1}=\operatorname{Tr}_{\mathscr{R}} t^{\left(c_{v} / 3\right)-J(0)}
$$

for the R-type SCA. The same property also holds for the NS-type SCA since there exists the $\mathrm{R} \leftrightarrow \mathrm{NS}$ isomorphism $\tilde{\sigma}_{-1 / 2}^{f}: B_{\hat{\Lambda}, \hat{\xi}}^{(0)} \cong B_{\hat{\Lambda}, \hat{\xi}}^{1 / 2}$.

The equality (5.12) can be read as a duality relation when we note the following inequality for the charges (5.7):

$$
0 \leqq Q(\chi) \leqq \frac{c_{v}}{3}, \quad \chi \in \mathscr{I},
$$

where $c_{v}$ is the central charge in (2.39). The above inequality follows from noting the positive definite operators $T(0) \geqq 0,\left\{G^{+}(-1), G^{-}(1)\right\}=2 T(0)-3 J(0)+\frac{2 c_{v}}{3} \geqq 0$ and the relation $\left\{G^{+}(0), G^{-}(0)\right\}=2 T(0)-J(0)=0$ on the space $\mathscr{R}$. (These properties do not depend on the types of the SCA, i.e., R or NS.)

(5-3) Grassmannian Model and etc. Here we present some calculations of the Poincaré polynomial using specific models. Then the $\mathscr{D}$ in (3.39) will become explicit and the quotient $\mathscr{I}$ will become clear.

Let us, first, restrict our argument to the model of Grassmannian, the simplest representative of the hermitian symmetric spaces;

$$
\operatorname{Gr}(n, m) \equiv \frac{S U(n+m)}{S U(n) \times S U(m) \times U(1)} .
$$

For this model, the subset $W^{1}$ of the classical Weyl group $W$ (the symmetric group $\left.\mathfrak{S}_{n+m}\right)$ is well-known [Kos]. So we have only to reveal the structure of the quotient $\mathscr{I}$.

Let $\varepsilon_{1}, \ldots, \varepsilon_{n+m}$ be the orthonormal bases for the fundamental representation of $S U(n+m)$. We take the set of the positive roots as $\Delta_{+}=\left\{\varepsilon_{i}-\varepsilon_{j} \mid 1 \leqq i<j \leqq n+m\right\}$ and the simple roots as $\alpha_{(i)}=\varepsilon_{i}-\varepsilon_{i+1},(1 \leqq i \leqq n+m-1)$ and $\alpha_{(0)}=\delta-\left(\varepsilon_{1}-\varepsilon_{n+m}\right)$. As for the subgroups $S U(n)$ and $S U(m)$, we take $\Delta_{+}^{(n)}=\left\{\varepsilon_{i}-\varepsilon_{j} \mid 1 \leqq i<j \leqq n\right\}, \Delta_{+}^{(m)}=$ $\left\{\varepsilon_{a}-\varepsilon_{b} \mid n+1 \leqq a<b \leqq n+m\right\}$ and the simple roots $\Pi^{(n)}=\left\{\alpha_{(0), 1}=\delta_{1}-\left(\varepsilon_{1}-\varepsilon_{n}\right)\right.$, $\left.\alpha_{(i), 1}=\varepsilon_{i}-\varepsilon_{i+1}(1 \leqq i \leqq n-1)\right\}$, and $\Pi^{(m)}=\left\{\alpha_{(0), 2}=\delta_{2}-\left(\varepsilon_{n+1}-\varepsilon_{n+m}\right), \alpha_{(a), 2}=\varepsilon_{a}-\right.$ $\left.\varepsilon_{a+1}(n+1 \leqq a \leqq m+n-1)\right\}$, respectively. The root for $\mathfrak{m}_{+}$is $\Delta\left(\mathfrak{m}_{+}\right)=\left\{\varepsilon_{i}-\varepsilon_{a} \mid 1 \leqq\right.$ $i \leqq n, n+1 \leqq a \leqq n+m\}$. The subset $W^{1}$ consists of the elements $\sigma \in \Im_{n+m}$ such that

$$
\sigma^{-1}(1)<\sigma^{-1}(2)<\cdots<\sigma^{-1}(n), \quad \sigma^{-1}(n+1)<\sigma^{-1}(n+2)<\cdots<\sigma^{-1}(n+m) .
$$

The order $\left|W^{1}\right|$ is given by $(n+m) ! / n ! m !$.

According to the general theory, the diagram automorphisms are generated by $\rho_{(n+m)}=t_{\varepsilon_{1}} \omega_{\text {cyc. }}, \rho_{(n)}=t_{\varepsilon_{1}} \omega_{\text {cyc. }}^{(n)}, \rho_{(m)}=t_{\varepsilon_{n+1}} \omega_{\text {cyc. }}^{(m)}$ for $S U(n+m), S U(n)$ and $S U(m)$, respectively, where $\omega_{\text {cyc. }}=(1,2, \ldots, n+m), \omega_{\text {cyc. }}^{(n)}=(1,2, \ldots, n)$ and $\omega_{\text {cyc. }}^{(m)}=(n+1$, $n+2, \ldots, n+m)$. The diagram automorphisms $\rho_{(n)}$ and $\rho_{(m)}$ satisfy $\rho_{(n)}\left(\tilde{\Delta}_{+}^{(n)}\right)=\widetilde{\Delta}_{+}^{(n)}$ 
and $\rho_{(m)}\left(\tilde{\Delta}_{+}^{(m)}\right)=\tilde{\Delta}_{+}^{(m)}$ since they simply induce the permutations for the simple roots. Though each $\rho_{(n)}$ and $\rho_{(m)}$ is not the element of the Weyl group $\tilde{W}$ individually, we can make a combination $\sigma_{D}=\rho_{(n)} \rho_{(m)}^{-1}$ which belongs to the Weyl group. Then taking the conditions (3.34) and a) $\left.)^{\prime}-\mathrm{c}\right)^{\prime}$ below (3.36) for $\tilde{\mathfrak{D}}$ into account, we convince ourselves that, in this model, the elements of the group $\mathfrak{D}$ can be taken as

$$
\mathfrak{D}=\left\{1, \sigma_{D}, \ldots, \sigma_{D}^{d-1}\right\},
$$

where $d$ means the least common multiplier for $n$ and $m$.

Now let us define

$$
P(\mathrm{Gr}(n, m), \hat{\Lambda} ; t) \equiv \sum_{\omega^{1} \in W^{1}} t^{q\left(\hat{\Lambda}, \omega^{1}\right)}
$$

with $Q\left(\hat{\Lambda}, \omega^{1}\right) \equiv \frac{1}{k+g^{*}} q\left(\hat{\Lambda}, \omega^{1}\right)$. Using this polynomial, the Poincare polynomial may be expressed as

$$
P^{\prime}(\operatorname{Gr}(n, m), t)_{k}=\sum_{\hat{\lambda} \in P_{+. k}} P(\operatorname{Gr}(n, m), \hat{\Lambda} ; t)
$$

if there is no other field identification relation other than the one appearing in Proposition 5.3. However we easily notice that there is another identification relation which follows from the isomorphisms of the action $\gamma \in Q^{\perp}(\gamma \notin M)$. These isomorphisms are generated by the diagram automorphism $\rho_{\varepsilon_{1}}$ which is determined from the spectral flow $\sigma_{\varepsilon_{1}}$, and has order $n+m$. Thus we expect the correct Poincare polynomial is given by

$$
P(\mathrm{Gr}(n, m), t)_{k}=\frac{1}{n+m} P^{\prime}(\mathrm{Gr}(n, m), t)_{k} .
$$

We will soon find the cases that the denominator $n+m$ does not divide $P^{\prime}(\operatorname{Gr}(n, m), t)_{k}$. This phenomenon is the fixed points [LVW, Gep] remarked previously.

When we consider the case $k=1$, such a troublesome case does not occur. In this case the weight $\hat{\Lambda}$ of $\mathfrak{g}$-module can be written as $\hat{\Lambda}=\left(\lambda_{0}, \lambda_{1}, \ldots, \lambda_{n+m-1}\right)$ with only one of the $\lambda_{i}$ 's being equal to 1 and others zero. The diagram automorphism $\rho_{\varepsilon_{1}}$ induces the cyclic permutation of the Dynkin labels $\hat{\lambda}_{i}$. Therefore, making $\lambda_{0}=1$, the Poincaré polynomial can be written as

$$
P(\operatorname{Gr}(n, m), t)_{k=1}=\sum_{\omega^{1} \in W^{1}} t^{l\left(\omega^{1}\right)}
$$

and further, as is known,

$$
=\prod_{j=1}^{l} \frac{\left(1-t^{d_{j}(\mathfrak{g})+1}\right)}{\left(1-t^{d_{j}(\mathfrak{)})+1}\right)},
$$

where $d_{j}(\mathfrak{g})$ and $d_{j}(\mathfrak{h})$ are the exponents for $\mathfrak{g}$ and $\mathfrak{h}$, respectively. This relation holds also for other models with $k=1$ based on the simply laced hermitian symmetric spaces [LVW].

The phenomenon of the fixed point occurs when we consider the model $k \geqq 2$. To make the argument simple, but without loss of essential generality, let us restrict our attention to the model; $\operatorname{Gr}(2,2)$ at level $k=2$. In this case the naively expected 
Poincaré polynomial (5.20) is given by

$$
P^{\prime}(\operatorname{Gr}(2,2), t)_{k=2}=2\left(2 t^{8}+7 t^{6}+12 t^{4}+7 t^{2}+2\right) .
$$

We see that this polynomials is not divided by the order of the diagram automorphism; $n+m=4$. This can be understood as follows; the $k=2 \hat{\mathrm{g}}$-module consists of the three orbit of the diagram automorphism of $\hat{\mathrm{g}}:(2,0,0,0)$-orbit, $(1,1,0,0)$-orbit and $(1,0,1,0)$-orbit. Both the $(2,0,0,0)$-orbit and the $(1,1,0,0)$-orbit have order 4 , however the $(1,0,1,0)$-orbit has only order 2 . Therefore we must find the orbit with the fixed point, i.e. the orbit with length less than 4 among the orbit of the modules $B_{\hat{\Lambda}, \hat{\xi}_{\omega}}=B_{(1,0,1,0), \hat{\xi}_{\omega}}\left(\omega \in W^{1}\right)$. In fact, we can find that the modules $B_{\hat{\Lambda}_{,} \hat{\xi}_{\omega}}$ with $\left(\hat{\Lambda} ; \hat{\xi}_{\omega, 0}, \hat{\xi}_{\omega, 1}, \hat{\xi}_{\omega, 2}\right)=\left(\hat{\Lambda}_{1}+\hat{\Lambda}_{3} ; 6 \Lambda_{0}, 2 \hat{\Lambda}_{0}^{(1)}+2 \hat{\Lambda}_{1}^{(1)}, 2 \hat{\Lambda}_{0}^{(2)}+\hat{2}_{1}^{(2)}\right)$ and $\left(\hat{\Lambda}_{1}+\hat{\Lambda}_{3}\right.$; $\left.6 \Lambda_{0}-2 \rho_{2}, 2 \hat{\Lambda}_{0}^{(1)}+2 \hat{\Lambda}_{1}^{(1)}, 2 \hat{\Lambda}_{0}^{(2)}+2 \hat{\Lambda}_{1}^{(2)}\right)$ both represent the fixed point with order 2 with respect to the action $t_{\varepsilon_{1}+\varepsilon_{4}}$, where obvious notations for the fundamental weights are used. Taking this fact into account, we calculate the correct Poincaré polynomial as

$$
P(\operatorname{Gr}(2,2), t)_{k=2}=t^{8}+4 t^{6}+6 t^{4}+4 t^{2}+1 .
$$

As a next example for the model based on a non-simply laced Lie algebra, we briefly present the case of $\operatorname{Sp}(n) / S U(n) \times U(1)$. According to the convention in ref. [Bo], we adopt a normalization $(\theta, \theta)=4$. Then the system of the simple roots $\Pi, \Pi_{S}$ for $\operatorname{Sp}(n)$ and $S U(n)$ are $\Pi=\left\{\delta-2 \varepsilon_{1}, \varepsilon_{1}-\varepsilon_{2}, \ldots, \varepsilon_{n-1}-\varepsilon_{n}, 2 \varepsilon_{n}\right\}$ and $\Pi_{S}=$ $\left\{\delta-\left(\varepsilon_{1}-\varepsilon_{n}\right), \varepsilon_{1}-\varepsilon_{2}, \ldots, \varepsilon_{n-1}-\varepsilon_{n}\right\}$, respectively. The coroot lattice for $\operatorname{Sp}(n)$ is $Q^{\vee}=\mathbf{Z} \varepsilon_{1} \oplus \cdots \oplus \mathbf{Z} \varepsilon_{n}$. The classical Weyl group for $\operatorname{Sp}(n)$ is known as the semidirect product of the symmetric group $\mathfrak{S}_{n}$ and the actions $\varepsilon_{i} \rightarrow( \pm)_{i} \varepsilon_{i}$, and its order is $2^{2} n$ !. The element $\omega \in W^{1}$ can be constructed as follows; let $\sigma \in \mathfrak{\Im}_{n}$ such that

$$
\sigma(1)<\sigma(2)<\cdots<\sigma(s)=n, \quad \sigma(s+1)>\sigma(s+2)>\cdots>\sigma(n),
$$

with fixed $s(1 \leqq s \leqq n)$. Then we can take following elements as $\omega^{-1}$ for $\omega \in W^{1}$;

$$
\left(\begin{array}{cccccc}
1 & \cdots & s & s+1 & \cdots & n \\
\sigma(1) & \cdots & \pm \sigma(s) & -\sigma(s+1) & \cdots & -\sigma(n)
\end{array}\right),
$$

where the signs, $\pm \sigma(s)$, means the action $\varepsilon_{s} \rightarrow \pm \varepsilon_{\sigma(s)}$. In this case the group $\mathfrak{D}$ is generated by $\rho_{D}=t_{\varepsilon_{1}} \omega_{\text {cyc }}^{(n)} \in \tilde{W}$ which generates the diagram automorphism of order $n$ for the sub-algebra $s \hat{u}(\dot{n})$. The same arguments proceed as before and we obtain, for example,

$$
P(\operatorname{Sp}(3) / S U(3) \times U(1), t)_{k=1}=t^{6}+2 t^{5}+3 t^{4}+4 t^{3}+3 t^{2}+2 t+1 .
$$

In this case we cannot write (5.28) in the form as (5.23) even for $k=1$ case.

Returning to our initial problem in relation with the $\mathrm{L}-\mathrm{G}$ description for the $N=2$ Kazama-Suzuki models, we must mention that almost all models with level $k \geqq 2$ do not have the Poincare polynomial which can be reduced to the form (5.4). It is most probable that the global (non-perturbative) property of the theory makes it impossible to describe the theory in terms of a simple perturbative $\mathrm{L}-\mathrm{G}$ theory. Admitting that such a global effect of the theory can appear, it is quite obscure to what extent the global property appears in the description, what role the level $k$ plays, and $\cdots$. To reveal the geometrical meaning of the Poincaré polynomial will 
shed light on these problems; more precisely, to investigate the relation to the semi-infinite flag manifold from a mathematical viewpoint and to interpret our cohomology theory via topological field theories [W] from a physical viewpoint.

\section{Summary and Discussions}

We have studied in detail a cohomological structure behind the $N=2$ SCFT constructed by means of the affine Lie algebra pairs $(\hat{\mathfrak{g}}, \hat{\mathfrak{h}})$. We have formulated our cohomology theory as the affinization of the Lie algebra cohomology theory by Kostant. Kostant's classical Lie algebra cohomology theory can be recovered through a certain classical limit of our theory. The characteristic features that we have recognized in our affine Lie algebra case are the essential roles played by the Dynkin diagram automorphisms for the affine Lie algebras. Motivated by the Landau-Ginzburg description for the $N=2 \mathrm{SCFT}$, we have formulated the algebra automorphisms for $N=2 \mathrm{SCA}$, spectral flow, and then defined a Poincare polynomial associated with each $N=2 \mathrm{SCFT}$. This Poincaré polynomial might have some mathematical importance, e.g., this polynomial might represent a certain deformation of the Poincaré polynomial of the semi-infinite flag manifold $L G / L H$ [FF] for loop groups $L G$ and $L H$ through the introduction of a certain periodicity associated with the group $T_{0}$. The geometrical meaning of the Poincaré polynomial should be clarified in future investigations.

As for the $\mathrm{L}-\mathrm{G}$ description for the $N=2 \mathrm{SCFT}$ constructed by GKO construction, we must check as a first step for that purpose whether the Poincare polynomial we have defined can be written in the form of $P_{J}(t)$ in (5.4). We have found, contrary to our expectation, that there are many models whose Poincare polynomial cannot be written in the form (5.4). On this respect, there are arguments [LVW, Mar] that such theories are described by the orbifold $\mathrm{L}-\mathrm{G}$ theory. We must leave the detailed analysis to future investigations.

In any way, there seems not to exist a simple description in terms of the $\mathrm{L}-\mathrm{G}$ Lagrangian for the general $N=2$ SCFTs based on the GKO construction. And so, generally, we cannot expect a beautiful correspondence between the L-G theory with $N=2$ supersymmetry and the singularity theory as have been observed for the minimal theories with $c_{v}<3$. However it seems interesting and important to identify the $N=2$ SCFTs which are described by the known singularity types other than the simple singularity, $\mathrm{A}-\mathrm{D}-\mathrm{E}$.

Acknowledgements. One of the authors (S. H.) would like to express his gratitude to Y. Namikawa and Y. Yamada for valuable comments and discussions. He also thanks K. Hasegawa for useful discussions. This work is supported in part by Grant-in-Aid for Scientific Research from the Ministry of Education, Science and Culture of Japan No. 01790194.

\section{References}

[AGV] Arnold, V. I., Gusein-Zade, S. M., Varchenko, A. N.: Singularities of differentiable maps. I.II. Berlin: Birkhäuser 1988

[BDFM] Banks, T., Dixon, L. J., Martinec, E.: Nucl. Phys. B299, 613 (1988) 
[BGG] Bernstein, I. N., Gelfand, I. M., Gefland, S. I.: Differential operators on the base affine space and a study of $\mathfrak{g}$-modules. In: Lie group and their representations, Gelfand, I. M. (ed.) New York: Wiley 1975

[Bo] Bourbaki, N.: Groups et algégre de Lie (Chaps. 7 and 8). Pairs: Herman 1975

[BPZ] Belavin, A. A., Polyakov, A. M., Zamolodchikov, A. B.: Infinite conformal symmetry in two-dimensional quantum field theories. Nucl. Phys. B241, 333 (1984)

[CIZ] Cappeli, A., Itzykson, C., Zuber, J.-B.: The A-D-E classification of minimal and $A_{1}^{(1)}$ conformal invariant theories. Commun. Math. Phys. 113, 1 (1987)

[DPZ] DiVeccia, P., Pertersen, J. L., Zheng, H. B.: Explicit construction of the $N=2$ superconformal algebra. Phys. Lett. B162, 327 (1985)

[FF1] Feigin, B. L., Frenkel, E.: Affine Kac-Moody algebras and bosonization. In: Physics and Mathematics of Strings. Knizhnik, V. Memorial Volume. Brinks, L. et al. (eds.) pp. 271-316. Singapore: World Scientific (1989)

[FF2] Feigin, B. L., Frenkel, E.: Affine Kac-Moody algebras and semi-infinite flag manifolds. Commun. Math. Phys. 128, 161 (1990)

[Fr] Frenkel, L. B.: Spinor representation of affine Lie algebra. Proc. Nat. Acad. Sci. USA 77, 6303 (1980)

[BFK] Boucher, W., Friedan, D., Kent, A.: Determinant formulas and unitarity for the $N=2$ superconformal algebras in two dimensions or exact results on string compactification. Phys. Lett. B172, 316 (1986)

[GL] Garland, H., Lepowski, J.: Lie algebra homology and the Macdonald-Kac formula. Inv. Math. 34, 34 (1976)

[Gep] Gepner, D.: Field identification in coset conformal field theories. Phys. Lett. B222, 207 (1989)

[GKO] Goddard, P., Kent, A., Olive, D.: Unitary representations of the Virasoro and super Virasoro algebras. Commun. Math. Phys. 103, 105 (1986)

[GO] Goddard, P., Olive, D.: Kac-Moody and Virasoro algebras in relation to quantum physics. Int. J. Mod. Phys. A1, 304 (1986)

[Has] Hasegawa, K.: Spin modules versions of Weyl's reciprocity theorem for classical Kac-Moody Lie algebras - an application to branching rule duality. Publ. RIMS, Kyoto Univ. 25, 741 (1989)

[IM] Iwahori, N, Matsumoto, H.: On some Bruhat decomposition and the structure of the Hecke rings of $p$-adic Chevalley group. Publ. Math. IHES 25, 5 (1965)

[Kac] Kac, V. G.: Infinite-dimensional Lie algebras. London/New York: Cambridge University Press 1985

[Kat] Kato, A.: Classification of modular invariant partition functions in two dimension. Mod. Phys. Lett. A2, 585 (1987)

[Kos] Kosant, B.: Lie algebra cohomology and the generalized Borel-Weil theorem. Ann. Math. 74, 329 (1961)

[KP] Kac, V. G., Perterson, P. H.: Infinite dimensional Lie algebra, theta-functions and modular forms. Adv. Math. 53, 125 (1984)

[KS] Kazama, Y., Suzuki, H.: New $N=2$ superconformal field theories and superstring compactification. Nucl. Phys. B321, 232 (1989); Characterization of $N=2$ superconformal models generated by the coset space method. Phys. Lett. B216, 112 (1989)

[Kum] Kumar, S.: Geometry of Schubert cells and cohomology of Kac-Moody algebra. J. Diff. Geom. 20, 389 (1984)

[KW] Kac. V. G., Wakimoto, M.: Modular and conformal invariance constraints in representation theory of affine algebras. Adv. Math. 70, 156 (1988)

[Mar] Martinec, E.: Criticality, catastrophes and compactifications. In: The Kniznik memorial volume. Brinks, L. et al. (eds.): World Scientific (1989)

[MS1] Moore, G., Seiberg, N.: Classical and quantum conformal field theory. Commun. Math. Phys. 123, 177 (1989)

[MS2] Moore, G., Seiberg, N.: Taming the conformal zoo. Phys Lett. 220B, 422 (1989)

[Na] Nahm, S: The Kac formula for the $N=1$ and $N=2$ superconformal algebras. Phys. Lett. B172, 323 (1986)

[SY] Schellekens, A. N., Yankielowwicz, S.: Field identification fixed points in the coset construction. Nucl. Phys. B334, 67 (1990) 
[Sug] Sugawara, H.: A field theory of currets. Phys. Rev. 170, 1659 (1968)

[TK] Tsuchiya, A., Kanie, R.: Unitary representations of the Virasoro algebra and branching law of representations of affine Lie algebra. Taniguchi Symp. PMMP, Katata 385 (1985)

[LVW] Lerche, W., Vafa, C., Warner, N. P.: Chiral rings in $N=2$ superconformal theories. Nucl. Phys. B324, 427 (1989)

[Ve] Verlinde, E.: Nucl. Phys. B300 [FS22], 360 (1988)

[VW] Vafa, C., Warner, N. P.; Catastrophes and the classification of conformal theories. Phys. Lett. B218, 51 (1989)

[W] Witten, E.: Quantum field theory and Jones polynomial. Commun. Math. Phys. 121, $351(1989)$

[Zam] Zamolodchikov, A. B.: Conformal symmety and multicritical points in two-dimensional quantum field theory. Sov. J. Nucl. Phys. 44, 529 (1986)

Communicated by $\mathrm{H}$. Araki 